\title{
Heavy holographic exotics: Tetraquarks as Efimov states
}

\author{
Yizhuang Liu* \\ Tsung-Dao Lee Institute, Shanghai Jiao University, Shanghai 200240, China \\ Maciej A. Nowak ${ }^{\dagger}$ \\ M. Smoluchowski Institute of Physics and Mark Kac Center for Complex Systems Research, \\ Jagiellonian University, 30-348 Kraków, Poland \\ Ismail Zahed \\ Department of Physics and Astronomy, Stony Brook University, Stony Brook, New York 11794-3800, USA
}

(Received 24 October 2019; published 24 December 2019)

\begin{abstract}
We provide a holographic description of nonstrange multiquark exotics as compact topological molecules by binding heavy-light mesons to a tunneling configuration in D8-D $\overline{8}$ that is homotopic to the vacuum state with a fixed Chern-Simons number. In the tunneling process, the heavy-light mesons transmute to fermions. Their binding is generic and arises from a trade-off between the dipole attraction induced by the Chern-Simons term and the U(1) fermionic repulsion. In the heavy quark limit, the openflavor tetraquark exotics $Q Q \bar{q} \bar{q}$ and $\bar{Q} \bar{Q} q q$ emerge as bound Efimov states in a degenerate multiplet $I J^{\pi}=\left(00^{+}, 01^{+}\right)$with opposite intrinsic Chern-Simons numbers $\pm \frac{1}{2}$. The hidden-flavor tetraquark exotics such as $Q \bar{Q} q \bar{q}, Q Q \bar{Q} \bar{q}$, and $Q Q \bar{Q} \bar{Q}$ as compact topological molecules are unbound. Other exotics are also discussed.
\end{abstract}

DOI: $10.1103 /$ PhysRevD.100.126023

\section{INTRODUCTION}

Several experimental collaborations [1-4] have reported new multiquark exotic states such as the neutral $X(3872)$ and the charged $Z_{c}(3900)^{ \pm}$and $Z_{b}(10610)^{ \pm}$, a priori outside the canonical quark model classification. More recently, the $\mathrm{LHCb}[4]$ has reported new pentaquark states $P_{c}^{+}(4380)$ and $P_{c}^{+}(4450)$ through the decays $\Lambda_{b}^{0} \rightarrow$ $J \Psi p K^{-}, J \Psi p \pi^{-}[5]$ and five narrow and neutral excited $\Omega_{c}^{0}$ baryon states that decay primarily to $\Xi_{c}^{+} K^{-}[6]$.

Some of the reported hidden-flavor tetraquark exotics appear to be loosely bound hadronic molecules of two heavy-light mesons [7-15], although other explanations for their composition are also suggested in Refs. [16-19]. The first estimates of the open-flavor and compact tetraquark exotics were made in the context of the bag model [20] and the random instanton model with full chiral and heavy

\footnotetext{
*yizhuang.liu@sjtu.edu.cn maciej.a.nowak@uj.edu.pl

*ismail.zahed@stonybrook.edu
}

Published by the American Physical Society under the terms of the Creative Commons Attribution 4.0 International license. Further distribution of this work must maintain attribution to the author(s) and the published article's title, journal citation, and DOI. Funded by SCOAP. quark symmetry in Refs. [21,22], in line with recent estimates using constituent quark models [23].

The reported pentaquark states with hidden charm initially suggested in Ref. [24] have been addressed by many [25-29] and include the newly reported neutrals $\Omega_{c}^{0}$ as discussed in Refs. [30-33]. Given the difficulty of tracking QCD in the infrared, it is not easy to identify a first principle mechanism for the formation of these multiquark states.

Most of the multiquark states reported so far involve both heavy and light quarks but fall outside the realm of the canonical quark model [23]. It is well established that the light quark sector of QCD exhibits spontaneous chiral symmetry breaking, while the heavy quark sector is characterized by heavy quark symmetry [34]. Both symmetries are at the origin of the chiral doubling suggested in heavy-light mesons [35,36] and confirmed experimentally in Refs. [37,38]. It is therefore important that a theoretical approach to the multiquark states should have manifest chiral and heavy quark symmetry, a clear organizational principle in the infrared, and should address concisely the multibody bound state problem.

The holographic principle in general [39-41] and the D4-D8-D $\overline{8}$ holographic setup in particular [42] provide a framework for addressing QCD in the infrared in the double limit of a large number of colors and strong coupling $\lambda=g_{\mathrm{YM}}^{2} N_{c}$. It is confining and exhibits spontaneous chiral 
symmetry breaking geometrically. In leading order in $1 / \lambda$, the light meson sector is well described by an effective action on the fused D8-D $\overline{8}$ branes that is consistent with known effective theories [43]. The same setup can be minimally modified to account for the description of heavy-light mesons as well, with full account of heavy quark symmetry [44]. Light and heavy-light baryons are dual to instantons and instanton-heavy meson bound states in bulk [44-50], providing a concise approach to the multibody bound state problem. In a way, the holographic construction provides a geometrical realization of the Skyrmion and its variants [26,51,52], without the shortcomings of the derivative expansion. Alternative holographic models for the description of heavy hadrons are developed in Refs. [53-55] without the dual strictures of chiral and heavy quark symmetry.

The organization of the paper is as follows. In Sec. II, we recall the geometrical setup for the derivation of the heavylight effective action with two light and one heavy flavors. We detail the heavy-light Dirac-Born-Infeld (DBI) action and the particular classical fields of interest for the description of the holographic multiquark states. In Sec. III, we derive explicitly a class of $\mathrm{O}(4)$ tunneling configurations with fixed Chern-Simons (CS) number in D8-D $\overline{8}$, that interpolate continuously between a unit topological charge (fermion) and zero topological charge (boson). We also derive their associated fermionic zero modes. In Sec. IV, we detail how a heavy meson attached to the tunneling configuration transmutes to a fermion. We also derive the pertinent Hamiltonian on the moduli associated to the topological molecule formed of heavy mesons attached to the $\mathrm{O}(4)$ tunneling configuration. In the heavy quark limit, the open-flavor and nonstrange tetraquarks and hexaquarks are found to be bound Efimov-like states. The hidden-flavor tetraquarks are not bound. In general, heavier exotics are not bound. Our conclusions are in Sec. V. We provide two Appendixes for completeness.

\section{HOLOGRAPHIC HEAVY-LIGHT EFFECTIVE ACTION}

\section{A. D-brane setup}

The D4-D8-D $\overline{8}$ setup for light flavor branes is standard [42]. The minimal modification that accommodates heavy mesons makes use of an extra heavy brane as discussed in Ref. [44]. It consists of $N_{f}$ light D8-D $\overline{8}$ branes (L) and one heavy $(\mathrm{H})$ probe brane in the cigar-shaped geometry that spontaneously breaks chiral symmetry. A schematic description of the setup for $N_{f}=2$ is shown in Fig. 1 . We assume that the L-brane world volume consists of $R^{4} \times S^{1} \times S^{4}$ with [0 - 9] dimensions. The light 8-branes are embedded in the $[0-3+5-9]$ dimensions and set at the antipodes of $S^{1}$ which lies in the fourth dimension. The warped [5-9]-space is characterized by a finite size $R$ and a horizon at $U_{K K}$.

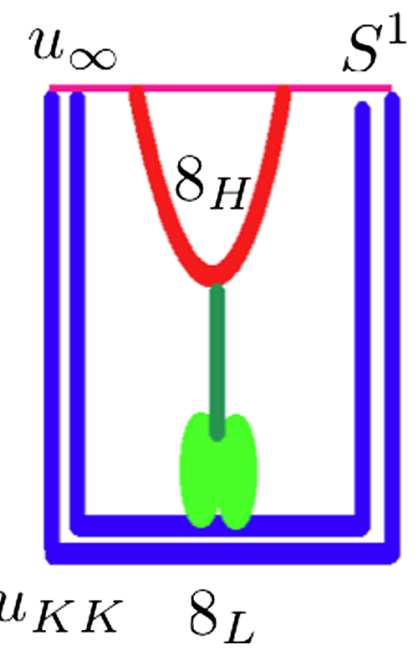

FIG. 1. $N_{f}=2$ antipodal $8_{L}$ light branes and one $8_{H}$ heavy brane shown in the $\tau U$ plane, with a bulk $\mathrm{O}(4)$ symmetric tunneling configuration with a turning point, embedded in $8_{L}$ and a massive HL string connecting them.

\section{B. DBI action}

The effective action on the probe L-branes consists of the non-Abelian DBI and CS action. After integrating over the $S^{4}$, the leading contribution in $1 / \lambda$ to the DBI action is

$$
S_{\mathrm{DBI}} \approx-\kappa \int d^{4} x d z \operatorname{Tr}\left(\mathbf{f}(z) \mathbf{F}_{\mu \nu} \mathbf{F}^{\mu \nu}+\mathbf{g}(z) \mathbf{F}_{\mu z} \mathbf{F}^{\nu z}\right) .
$$

The warping factors are

$$
\mathbf{f}(z)=\frac{R^{3}}{4 U_{z}}, \quad \mathbf{g}(z)=\frac{9}{8} \frac{U_{z}^{3}}{U_{K K}}
$$

with $U_{z}^{3}=U_{K K}^{3}+U_{K K} z^{2}$, and $\kappa \equiv a \lambda N_{c}$ and $a=1 /\left(216 \pi^{3}\right)$ [42]. Our conventions are $(-1,1,1,1,1)$ with $A_{M}^{\dagger}=-A_{M}$ and the labels $M, N$ running over $\mu, z$ only in this section. All units are given in terms of $M_{K K}=1$, which is readily recovered by dimensional inspection. The effective fields in the field strengths are [44]

$\mathbf{F}_{M N}=\left(\begin{array}{cc}F_{M N}-\Phi_{[M} \Phi_{N]}^{\dagger} & \partial_{[M} \Phi_{N]}+A_{[M} \Phi_{N]} \\ -\partial_{[M} \Phi_{N]}^{\dagger}-\Phi_{[M}^{\dagger} A_{N]} & -\Phi_{[M}^{\dagger} \Phi_{N]}\end{array}\right)$.

The matrix-valued 1-form gauge field is

$$
\mathbf{A}=\left(\begin{array}{cc}
A & \Phi \\
-\Phi^{\dagger} & 0
\end{array}\right)
$$

For $N_{f}=2$, the naive Chern-Simons 5 -form is

$$
S_{\mathrm{CS}}=\frac{i N_{c}}{24 \pi^{2}} \int_{M_{5}} \operatorname{Tr}\left(A F^{2}-\frac{1}{2} A^{3} F+\frac{1}{10} A^{5}\right) .
$$


We note that for only $N_{f}>2$ it fails to reproduce the correct transformation law under the combined gauge and chiral transformations [48]. In particular, when addressing the $N_{f}=3$ baryon spectra, Eq. (5) does not reproduce the important hypercharge constraint [48] but can be minimally modified to do that.

For $N_{f}$ coincidental branes, the $\Phi$ multiplet is massless, but for separated branes as illustrated in Fig. 1, they are massive with the additional contribution

$$
\frac{1}{2} m_{H}^{2} \operatorname{Tr}\left(\Phi_{M}^{\dagger} \Phi_{M}\right)
$$

The value of $m_{H}$ is related to the separation between the light and heavy branes, which is about the length of the HL string. Below, $m_{H}$ will be taken as the heavy meson mass.

\section{Light fields}

In the coincidental brane limit, light baryons are interchangeably described as a flavor instanton or a D4-brane wrapping the $S^{4}$. The instanton size is small with $\rho \sim 1 / \sqrt{\lambda}$ after balancing the order $\lambda N_{c}$ bulk gravitational attraction with the subleading and of order $\lambda^{0} N_{c} \mathrm{U}(1)$ induced topological repulsion [42].

To describe tetraquark states which carry zero topological charge or baryon number, but are still tightly bound by the underlying light gauge field in holography, we suggest using a tunneling configuration on the sphaleron path that is homotopic to the vacuum state. The configuration will carry a fixed Chern-Simons number. We will seek it using the maximally symmetric $\mathrm{O}(4)$ gauge field

$$
A_{M}(y)=-\left.\bar{\sigma}_{M N} \partial_{N} F(y) \quad F_{z m}(y)\right|_{|y|=R}=0,
$$

subject to the condition of zero "electric" field strength at the turning point $R=\rho$. From here on, $M, N$ runs only over $1,2,3, z$ unless specified otherwise. If $\rho \sim 1 / \sqrt{\lambda}$ is the typical size of these tunneling configurations, then it is natural to recast the DBI action using the rescaling

$$
\begin{aligned}
\left(x_{0}, x_{M}\right) & \rightarrow\left(x_{0}, x_{M} / \sqrt{\lambda}\right), \quad \sqrt{\lambda} \rho \rightarrow \rho \\
\left(A_{0}, A_{M}\right) & \rightarrow\left(A_{0}, \sqrt{\lambda} A_{M}\right) .
\end{aligned}
$$

The rescaled fields satisfy the equations

$$
D_{M} F_{M N}=0 \quad \partial_{M}^{2} A_{0}=-\frac{1}{32 \pi^{2} a} F_{a M N} \star F_{a M N}
$$

with the use of the Hodge dual notation, subject to the turning point condition (7) in leading order in $1 / \lambda$. The detailed solution to (9) will be given below. Unlike the instanton, which is stable, these tunneling configurations are unstable and tend to relax to the vacuum state. They are the $\mathrm{O}(4)$ analog of an instanton-anti-instanton configuration running to its demise through the valley. Below, we will show that they can stabilize quantum mechanically when heavy mesons bind to them.

\section{Heavy-light fields}

Let $\left(\Phi_{0}, \Phi_{M}\right)$ be the pair of heavy quantum fields that bind to the tunneling configuration above. If again $\rho \sim$ $1 / \sqrt{\lambda}$ is their typical size, then it is natural to recast the heavy-light part of the DBI action using the additional rescaling

$$
\left(\Phi_{0}, \Phi_{M}\right) \rightarrow\left(\Phi_{0}, \sqrt{\lambda} \Phi_{M}\right) .
$$

The interactions between the light gauge fields $\left(A_{0}, A_{M}\right)$ and the heavy-light gauge fields $\left(\Phi_{0}, \Phi_{M}\right)$ follow from their sharing of the light degrees of freedom in the form of a light brane as illustrated in Fig. 1, since $A \sim q \bar{q}$ and $\Phi \sim q \bar{Q}$. This is explicit in the matrix-valued form $\mathbf{A}$ in (4). All mixed interactions follow by inserting $\mathbf{A}$ in (1) and (5) and expanding as detailed in Ref. [44].

With this in mind, and to quadratic order, the interaction splits to several contributions [44]

$$
\mathcal{L}=a N_{c} \lambda \mathcal{L}_{0}+a N_{c} \mathcal{L}_{1}+\mathcal{L}_{C S}
$$

which are quoted in the Appendix for completeness. Here, we only need the leading contributions stemming from (11) in the additional heavy mass limit $m_{H} \rightarrow \infty$. For that, we split $\Phi_{M}=\phi_{M} e^{-i m_{H} x_{0}}$ for particles $\left(m_{H} \rightarrow-m_{H}\right.$ for antiparticles). The leading order contribution takes the form

$\mathcal{L}_{0}=-\frac{1}{2}\left|f_{M N}-\star f_{M N}\right|^{2}+2 \phi_{M}^{\dagger}\left(F_{M N}-\star F_{M N}\right) \phi_{N}$,

subject to the constraint equation $D_{M} \phi_{M}=0$ with

$$
f_{M N}=\partial_{[M} \phi_{N]}+A_{[M} \phi_{N]},
$$

while the subleading contributions in (11) to order $\lambda^{0} m_{H}$ simplify to

$$
\begin{aligned}
\frac{\mathcal{L}_{1}}{a N_{c}} & \rightarrow 4 m_{H} \phi_{M}^{\dagger} i D_{0} \phi_{M} \\
\mathcal{L}_{\mathrm{CS}} & \rightarrow \frac{m_{H} N_{c}}{16 \pi^{2}} \phi_{M}^{\dagger} \star F_{M N} \phi_{N} .
\end{aligned}
$$

For self-dual light gauge fields with $F_{M N}=\star F_{M N}$, the last contribution in (12) vanishes, and the minimum is reached for $f_{M N}=\star f_{M N}$. This observation when combined with the transversality condition for $D_{M} \phi_{M}=0$ amounts to a first order equation for the combination $\psi=\bar{\sigma}_{M} \phi_{M}$ with $\sigma_{M}=(i, \vec{\sigma})$, i.e.,

$$
\sigma_{M} D_{M} \psi=D \psi=0
$$


as noted in Ref. [44]. In a self-dual gauge configuration, the heavy spin-1 meson transmutes to a massless spin- $-\frac{1}{2}$ spinor that is Bogomol'nyi-Prasad-Sommerfield bound in leading order. For the tunneling configuration in (7), the selfduality condition no longer holds. With this in mind, we now proceed to determine first the explicit tunneling configuration $\left(A_{0}, A_{M}\right)$ by solving (9) and then its variational zero mode.

\section{TUNNELING FIELD AND ITS FERMIONIC ZERO MODE}

In this section, we detail the construction of a family of $\mathrm{O}(4)$ symmetric tunneling configurations of the type (7) that are solution to (9). They carry a fractional ChernSimons number at the turning point and interpolate parametrically between the instanton and the sphaleron configuration at the turning point when continued to Minkowski space. Their O(3) symmetric relatives through a conformal transformation are discussed in the Appendix. We note that similar configurations were used in the context of explosive sphalerons and their applications to finite energy collisions [56-58]. We also derive their corresponding fermionic zero modes, which will prove useful for the discussion of the heavy-meson bound states through transmutation.

\section{A. O(4) tunneling configuration}

Consider the $\mathrm{O}(4)$ static and symmetric ansatz for the $\mathrm{SU}(2)$ gauge configuration

$$
A_{a M}(y)=2 \bar{\eta}_{a M N} \frac{y_{N}}{y^{2}} f(\xi)
$$

with the conformal variable $\xi(y)=\frac{1}{2} \ln \left(y^{2} / \rho^{2}\right)$. The antiinstanton and antisphaleron configurations follow from a similar construction with a dual background $\bar{\eta} \rightarrow \eta$. In terms of (16), the static $\mathrm{O}(4)$ symmetric part of the YangMills action in (1) without warping reads

$$
\begin{aligned}
S_{\mathrm{DBI}} & \rightarrow-\kappa T \int d^{3} x d z F_{a M N} F_{a M N} \\
& =+\frac{24 \pi^{2} T}{g_{\kappa}^{2}} \int d \xi\left(\frac{f^{\prime 2}(\xi)}{2}+V(f(\xi))\right),
\end{aligned}
$$

with $T$ the length of time, the induced effective coupling for the flavor gauge fields $g_{\kappa}^{2}=1 / \kappa$, and the double well potential

$$
V(f)=2(f \bar{f})^{2},
$$

with $\bar{f}=1-f$. The $\mathrm{O}(4)$ profile $f(\xi)$ extremizes (17) by satisfying

$$
\frac{d^{2} f}{d \xi^{2}}=4\left(f^{2}-f\right)(2 f-1)
$$

which is of the Jacobi type. Remarkably, the solution to (19) with a sphaleronlike turning point at $\xi=0$ with $f^{\prime}(\xi=0)=0$ can be found explicitly

$f_{k}(\xi)=\frac{1}{2}\left(1+\left(\frac{2 k^{2}}{1+k^{2}}\right)^{\frac{1}{2}} \operatorname{sn}\left(\xi\left(\frac{2}{1+k^{2}}\right)^{\frac{1}{2}}, k\right)\right)$,

with sn the Jacobi sine function. We note that the solution (20) is $\xi$-periodic with period

$$
T_{k}=2 K(k)\left(\frac{1+k^{2}}{2}\right)^{\frac{1}{2}} .
$$

Her, $K(k)$ is the elliptic function, and $\xi \in\left[-\frac{T_{k}}{2}, \frac{T_{k}}{2}\right]$. In Fig. 2, we show (20) for $k=0.1,0.5,1$.

The parameter $k$ relates to the sphaleronlike energy at the turning point

$$
E_{k}=\frac{24 \pi^{2}}{g_{\kappa}^{2}} V\left(f_{k}(\xi=0)\right)=\frac{3 \pi^{2}}{g_{\kappa}^{2}}\left(\frac{1-k^{2}}{1+k^{2}}\right)^{2} .
$$

At $k=0$, we recover the expected sphaleron energy $E_{0}=3 \pi^{2} / g_{\kappa}^{2}$ with the constant profile $f_{0}(\xi)=\frac{1}{2}$. At the instanton point $k=1$ with zero energy $E_{1}=0$, we recover the instanton interpolating profile

$$
f_{1}(\xi)=\frac{1}{2}+\frac{1}{2} \operatorname{sn}(\xi, 1)=\frac{e^{2 \xi}}{1+e^{2 \xi}}
$$

as illustrated in Fig. 2. In general, the solution (20) carries Chern-Simons number $N_{k}$ and energy $E_{k}$ at the turning point, that are tied through the profile of the potential (18)

$$
N_{k}\left(1-N_{k}\right)=\frac{1}{4}\left(\frac{E_{k}}{E_{0}}\right)^{\frac{1}{2}}
$$

with $N_{1}=1$ the instanton topological charge and $N_{0}=\frac{1}{2}$ the sphaleron Chern-Simons number. Only the solution with $N_{1}=1$ is self-dual. All the other configurations with $N_{k}<1$ are extrema rather than minima, and therefore prone to decay. They are homotopic to the vacuum state.

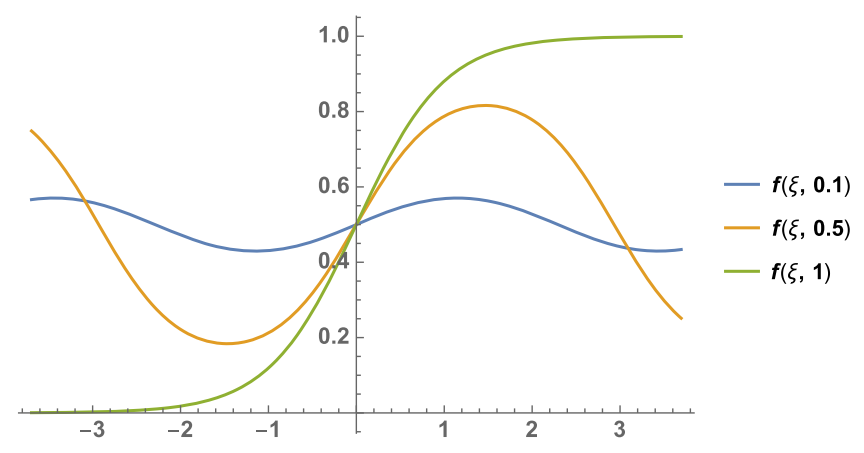

FIG. 2. $f_{k}(\xi)$ for $k=0.1,0.5,1$. 


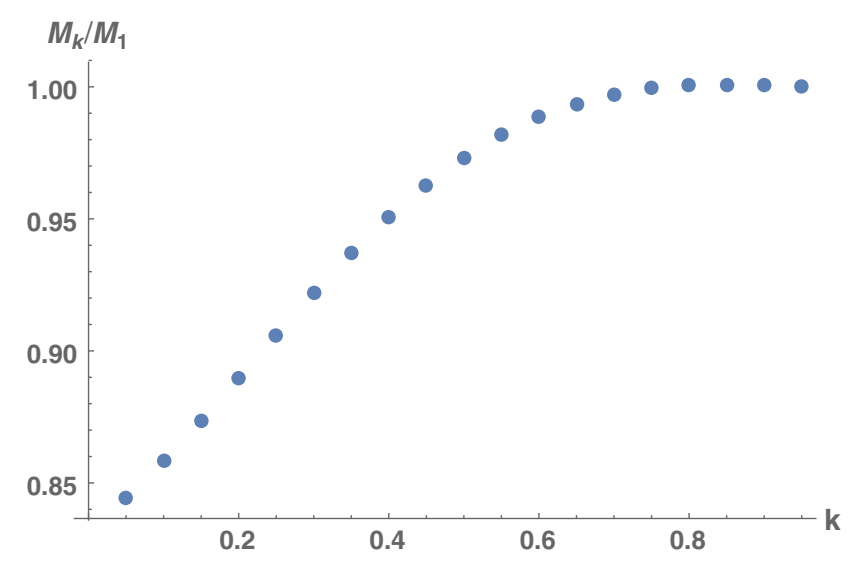

FIG. 3. The mass ratio $M_{k} / M_{1}$ vs k.

For the holographic dual hadronic configurations, the more relevant quantity is the action (17) for the generalized tunneling configurations (20). Since the solutions are periodic reflecting on the periodicity of the sphaleron ridge, we have for the fundamental period the action

$$
S_{k}=\frac{24 \pi^{2} T}{g_{\kappa}^{2}} \int_{0}^{T_{k}} d \xi\left(\frac{f^{\prime 2}(\xi)}{2}+V(f(\xi))\right),
$$

which gives $S_{1}=\left(8 \pi^{2} / g_{\kappa}^{2}\right) T$ at the instanton point as expected, and

$$
S_{0}=\frac{3 \pi^{2}}{g_{\kappa}^{2}} T_{0} T=\frac{3 \pi^{3}}{\sqrt{2} g_{\kappa}^{2}} T
$$

at the sphaleron point. In particular, the holographic mass of the tunneling configuration without warping can be read from (25) as $M_{k}=S_{k} / T$. We note that the holographic mass ratio at the sphaleron to instanton point is $M_{0} / M_{1}=$ $3 \pi / 8 \sqrt{2}<1$. In Fig. 3 , we show the mass ratio $M_{k} / M_{1}=$ $S_{k} / S_{1}$ for different values of $0 \leq k \leq 1$.

\section{B. U(1) gauge field}

The U(1) gauge field in (9) is sourced by the local topological charge density carried by the $\mathrm{O}(4)$ tunneling configuration (16). For that, it is useful to determine the field strength $F_{M N}$ and its dual $\star F_{M N}$, or more explicitly the electric and magnetic fields

$$
\begin{aligned}
y^{2} \vec{E}= & \vec{\sigma}\left(f^{\prime}-\frac{(\vec{y})^{2}}{y^{2}}\left(f^{\prime}-2 f \bar{f}\right)\right) \\
& +\frac{\left(\vec{y} \cdot \vec{\sigma} \vec{y}-y_{4} \vec{\sigma} \times \vec{y}\right)}{y^{2}}\left(f^{\prime}-2 f \bar{f}\right) \\
y^{2} \vec{B}= & \vec{\sigma}\left(2 f \bar{f}+\frac{(\vec{y})^{2}}{y^{2}}\left(f^{\prime}-2 f \bar{f}\right)\right) \\
& -\frac{\left(\vec{y} \cdot \vec{\sigma} \vec{y}-y_{4} \vec{\sigma} \times \vec{y}\right)}{y^{2}}\left(f^{\prime}-2 f \bar{f}\right),
\end{aligned}
$$

with $E^{i}=F^{i 4}$ and $B^{i}=\frac{1}{2} \epsilon^{i j k} F^{j k}$. For self-dual fields, $f^{\prime}=$ $2 f \bar{f}$ and $\vec{E}=\vec{B}$ as expected for the instanton path. The $\mathrm{U}(1)$ field satisfies

$$
\partial_{M}^{2} A_{0}=-\frac{3}{4 \pi^{2} a y^{4}}\left(2 f^{\prime} f \bar{f}\right),
$$

which can be inverted if we define

$$
A_{0}(y)=\frac{1}{y^{2}} \phi_{1}(\xi)
$$

so that $\phi_{1}(\xi)$ is the solution to

$$
\begin{aligned}
\phi_{1}^{\prime \prime}-2 \phi_{1}^{\prime} & =-\frac{3}{4 \pi^{2} a}\left(2 f^{\prime} f \bar{f}\right) \\
& \equiv F_{0}(\xi, k) \equiv-\frac{3}{4 \pi^{2} a} \mathbb{F}_{0}(\xi, k)
\end{aligned}
$$

which is sourced by the topological charge density. The net topological charge is

$$
Q_{\mathrm{top}}(k)=3 \int_{-\frac{T_{k}}{2}}^{\frac{T_{k}}{2}} d \xi \mathbb{F}_{0}(\xi, k)
$$

Note that $\mathbb{F}_{0}(\xi, k)$ is a total derivative as it should be,

$$
\mathbb{F}_{0}(\xi, k)=\left(f^{2}-\frac{2}{3} f^{3}\right)^{\prime}
$$

which is identically zero at the sphaleron point since $f_{0}=\frac{1}{2}$. Equation (31) is monotonous in $k$ as shown in Fig. 4. It interpolates continuously between the sphaleron path at $k=0$ with zero topological charge and the instanton path at $k=1$ with unit topological charge.

Equation (30) is readily solved using

$$
\phi_{1}(\xi)=C_{1}+C_{2} e^{+2 \xi}+\frac{1}{2} \int_{-\frac{T_{k}}{2}}^{\xi} d \xi_{1}\left(e^{+2 \xi-2 \xi_{1}}-1\right) F_{0}\left(\xi_{1}, k\right) .
$$

The constants of integration $C_{1,2}$ can be fixed by choosing the solution to satisfy the zero boundary condition at $\xi=-\frac{T_{k}}{2}$ and the regular boundary condition at $\xi=\frac{T_{k}}{2}$. This means that

$$
-C_{2}=e^{T_{k}} C_{1}=\frac{1}{2} \int_{-\frac{T_{k}}{2}}^{\frac{T_{k}}{2}} e^{-2 \xi_{1}} F_{0}\left(\xi_{1}, k\right) d \xi_{1},
$$




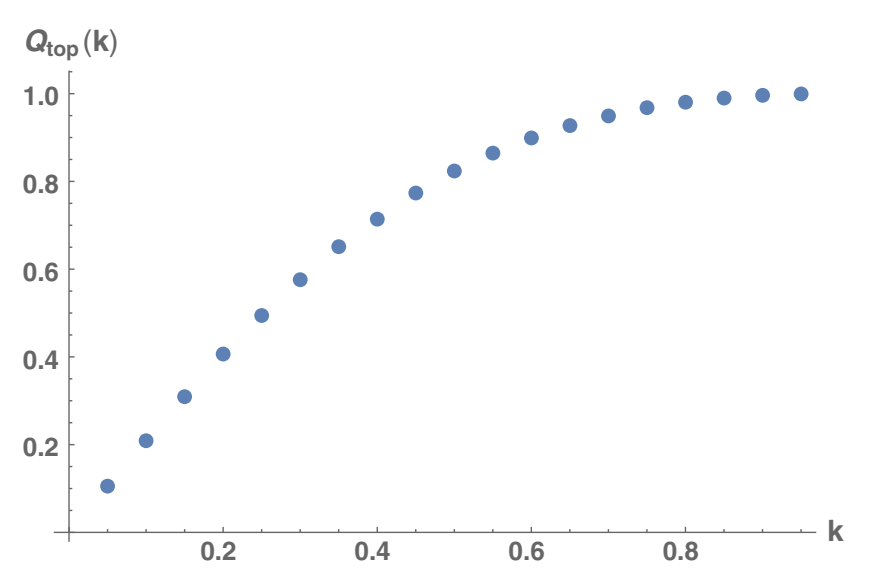

FIG. 4. Topological charge vs k.

which explicitly gives

$$
\begin{aligned}
\phi_{1}(\xi)= & +\frac{1}{2} \int_{-\frac{T_{k}}{2}}^{\xi} d \xi_{1}\left(e^{+2 \xi-2 \xi_{1}}-1\right) F_{0}\left(\xi_{1}, k\right) \\
& -\frac{1}{2}\left(e^{2 \xi}-e^{-T_{k}}\right) \int_{-\frac{T_{k}}{2}}^{\frac{T_{k}}{2}} e^{-2 \xi_{1}} F_{0}\left(\xi_{1}, k\right) d \xi_{1} .
\end{aligned}
$$

\section{O(4) fermionic zero mode}

The zero mode solution in the $\mathrm{O}(4)$ configuration (1) solves the Dirac equation

$$
\left(\partial_{M}-i A_{M}\right) \gamma_{M} \psi=0 .
$$

In the chiral basis with spin matrices $\bar{\sigma}_{M s}=\left(1,-i \vec{\sigma}_{s}\right)$ and

$$
\gamma_{5}=\left(\begin{array}{cc}
1 & 0 \\
0 & -1
\end{array}\right) \quad \gamma_{\mu}=\left(\begin{array}{cc}
0 & \sigma_{M s} \\
\bar{\sigma}_{M s} & 0
\end{array}\right),
$$

Eq. (36) splits into two chirality modes $\psi_{ \pm}$, each the conjugate of the other. Note the difference between the preceding conventions and the Hermiticity of the gauge field in this section only. If we note that the 't Hooft symbol satisfies the color identity

$$
\bar{\sigma}_{M c} \sigma_{N c}=\sigma_{a c} \bar{\eta}_{a M N}
$$

with the color matrices $\sigma_{M c}=\left(1,-i \vec{\sigma}_{c}\right)$, then the positive chirality mode associated to (36) satisfies

$$
\left(\bar{\sigma}_{M s} \partial_{M}+\frac{1}{2} \bar{\sigma}_{N s} \bar{\sigma}_{N c} \sigma_{M c} \partial_{M} F\right) \psi_{+}=0
$$

with the spin and color matrices commuting and $F(\xi)$ following from (16)

$$
F(\xi(y))=2 \int_{0}^{\xi(y)} d \xi^{\prime} f\left(\xi^{\prime}\right) .
$$

Note that, in writing (39), we have added a U(1) part to the gauge field for notational simplicity. It will be removed in the final step below. Equation (39) can be solved formally using

$$
\psi_{+}=\varphi \chi_{Q} \quad\left(\chi_{Q}\right)_{a \mu}=\epsilon_{a \mu},
$$

which is a singlet in color-spin space that satisfies

$$
\sigma_{M s} \chi_{Q}=\bar{\sigma}_{M c} \chi_{Q} \quad \bar{\sigma}_{M s} \chi_{Q}=\sigma_{M c} \chi_{Q} .
$$

Using (41)-(42) in (39) yields

$$
\left(\bar{\sigma}_{M s} \partial_{M}+\frac{1}{2} \bar{\sigma}_{N s} \sigma_{N s} \bar{\sigma}_{M s} \partial_{M} F\right) \varphi \chi_{Q}=0 .
$$

It is here that we need to remove the U(1) contribution noted above through the substitution

$$
\bar{\sigma}_{N s} \sigma_{N s} \chi_{Q}=\left(1+\left(\vec{\sigma}_{s}\right)^{2}\right) \chi_{Q} \rightarrow\left(\vec{\sigma}_{s}\right)^{2} \chi_{Q}=3 \chi_{Q}
$$

leading to the $\mathrm{O}(4)$ symmetric equation for the zero mode amplitude

$$
\varphi^{\prime}+\frac{3}{2} F^{\prime} \varphi=0
$$

The spinor zero mode of positive parity is

$$
\psi_{+}(y)=\mathbf{C} e^{-\frac{3}{2} F(\xi(y))} \chi_{Q} \equiv f_{0}(y) \chi_{Q},
$$

with the normalization constant fixed within $T_{k}$,

$$
\mathbf{C}=\left|\int_{T_{k}} d^{4} y e^{-3 F(\xi(y))}\right|^{-\frac{1}{2}} .
$$

\section{MULTIQUARK EXOTICS AS TOPOLOGICAL MOLECULES}

In the triple limit of a large number of colors $N_{c}$, strong coupling $\lambda$, and heavy meson mass $m_{H}$, the holographic multiquark exotics can be constructed by attaching to the $\mathrm{O}(4)$ tunneling gauge configuration an arbitrary number of heavy-light mesons. Of course, in reality, only a few can stick. The fermionic repulsion induced through a U(1) coupling to the Chern-Simons term stabilizes the tunneling configurations viewed as an instantonanti-instanton process.

\section{A. Heavy bound meson}

For $k=1$ with net topological charge 1, the heavy meson field in the self-dual classical background (7) transmutes to a fermionic zero mode (15) as initially noted in Ref. [44]. For $k<1$, which is the case of interest with fractional topological charge (fixed Chern-Simons 
number), the classical background (7) is no longer selfdual, and the minimum of (12) can be solved using a variational fermionic ansatz of the type (15)

$$
\phi_{M} \rightarrow \bar{\sigma}_{M} \psi_{+} \equiv \bar{\sigma}_{M} g(r) \chi_{Q},
$$

with the radial coordinate $r=|y|$. In terms of (48), the leading contribution to the action $\mathcal{S}_{0}$ associated to $\mathcal{L}_{0}$ in (11) is

$$
\begin{aligned}
\mathcal{S}_{0} & =-\int d^{4} y\left(f_{M N}^{\dagger} f_{M N}+2 \phi_{M}^{\dagger} F_{M N} \phi_{N}\right) \\
& =-\int 2 \pi^{2} d r\left(6 \mathbb{S}_{0}(g(r))\right) \chi_{Q}^{\dagger} \chi_{Q}
\end{aligned}
$$

with

$\mathbb{S}_{0}(g)=r^{3}\left(g^{\prime 2}+2 g g^{\prime} G+g^{2}\left(-2 G^{\prime}-\frac{6 G}{r}+9 G^{2}\right)\right)$.

Here, $g^{\prime}=d g / d r, G(r)=f / r$ and $G^{\prime}=d G / d r$ with the background gauge field expressed in the r-coordinate

$$
A_{M}=-\bar{\sigma}_{M N} \frac{y_{M}}{r} G(r) .
$$

The local minimum for $\mathbb{S}_{0}(g)$ requires that $g(r)$ satisfies

$$
g^{\prime \prime}+\frac{3}{5} g^{\prime}-\left(-3 G^{\prime}-\frac{9 G}{r}+9 G^{2}\right) g=0
$$

A special solution to (52) is of the form

$$
g(r)=e^{-3 \int_{0}^{r} d r^{\prime} G\left(r^{\prime}\right)}=e^{-\frac{3}{2} F(\xi)},
$$

which is readily seen to satisfy

$$
\begin{aligned}
g^{\prime}+3 G g & =0 \\
g^{\prime \prime}+3 G^{\prime} g+3 G g^{\prime} & =0
\end{aligned}
$$

and therefore

$$
\begin{gathered}
\left(g^{\prime}+3 G g\right)+\left(\frac{3}{r}-3 G\right)\left(g^{\prime \prime}+3 G^{\prime} g+3 G g^{\prime}\right) \\
=g^{\prime \prime}+\frac{3}{r} g^{\prime}+\left(-3 G^{\prime}-\frac{9 G}{r}+9 G^{2}\right) g=0
\end{gathered}
$$

which is (52). We note that for $k=1$ which corresponds to the instanton path Eq. (53) reduces to the standard fermionic zero mode $\left(r^{2}+\rho^{2}\right)^{-\frac{3}{2}}$. Equation (48) with (53) is transverse $D_{M} \phi_{M}=0$ for all values of $k$. In Ref. [44], $\chi_{Q} \rightarrow \chi_{Q}(t)$ describes the induced fermionic moduli upon binding, which is how the heavy quark of the original heavy-light meson manifests itself in this limit.

\section{B. Action for the topological molecule}

With the above in mind, and following the arguments presented in Ref. [44] (see Sec. VB), the pertinent contributions to the action for the topological molecule to order $\lambda^{0} m_{H}$ are

$$
\begin{aligned}
\frac{S_{k}}{a N_{c}} \approx & \int d t\left(\frac{M_{k}}{a N_{c}}-16 m_{H}^{2} \chi_{Q}^{\dagger} \chi_{Q}\right) \\
& +\int d t d^{4} y\left(16 m_{H} g^{2}(r) \chi_{Q}^{\dagger} i \partial_{t} \chi_{Q}\right. \\
& \left.+\frac{m_{H}}{8 a \pi^{2}} \epsilon_{M N P Q} \phi_{M}^{\dagger} F_{N P} \phi_{Q}\right)+S_{C}\left(A_{0}\right)
\end{aligned}
$$

with the U(1) Coulomb contribution

$$
\begin{aligned}
S_{C}\left(A_{0}=i \psi\right) \\
\quad=\int\left(\frac{1}{2}(\nabla \psi)^{2}+\psi\left(\rho_{0}[A]-16 m_{H} g^{2}(r) \chi_{Q}^{\dagger} \chi_{Q}\right)\right)
\end{aligned}
$$

due to the attraction induced by the Chern-Simons density $\rho_{0}(A)$ and the self-repulsion. More explicitly, we have

$S_{C}\left(A_{0}\right)=16 m_{H} \chi_{Q}^{\dagger} \chi_{Q} \int g^{2}(r)\left(-i A_{0}^{\mathrm{cl}}\right)-\alpha_{C}\left(16 m_{H} \chi_{Q}^{\dagger} \chi_{Q}\right)^{2}$

with the Coulomb factor

$$
\alpha_{C}=\frac{1}{2} \int g^{2}(r) \frac{1}{-\nabla^{2}} g^{2}(r) .
$$

The contribution to (56)

$$
-2 m_{H}\left(\phi_{0}^{\dagger} i D_{M} \phi_{M}-\text { c.c }\right)=0
$$

drops out for any $k$, thanks to the transversality of the zero mode (48). We recall that for $k=1$ the tunneling configuration is self-dual, but not otherwise. This configuration is an instanton with topological charge 1, and (56) describes the action of holographic heavy baryons [44].

\section{Hamiltonian for the topological molecule}

The molecular Hamiltonian associated to (56) follows using the canonical rules for $\chi_{Q}$ in the form

$$
\begin{aligned}
\mathbb{H}_{k} \approx & M_{k}+m_{H} \chi_{Q}^{\dagger} \chi_{Q}+\frac{\lambda \alpha_{0}(k)}{16 m_{H} \rho^{2}} \chi_{Q}^{\dagger} \chi_{Q}+\frac{3 \alpha_{1}(k)}{4 \pi^{2} a \rho^{2}} \chi_{Q}^{\dagger} \chi_{Q} \\
& +\frac{\alpha_{2}(k)}{8 \pi^{2} a N_{c} \rho^{2}}\left(\chi_{Q}^{\dagger} \chi_{Q}\right)^{2}
\end{aligned}
$$


after the rescaling of the fermionic fields

$$
\chi_{Q} \rightarrow \frac{1}{\left(16 a N_{c} m_{H}\right)^{\frac{1}{2}}} \chi_{Q} .
$$

Switching to the conformal coordinate $\xi$ and using the explicit profile for the tunneling gauge configuration $f(\xi)$ in (20), the U(1) gauge field $F_{0}(\xi, k)$ in (30), and the zero mode $g(\xi)$ in (53), we obtain for the k-dependent coefficients $\alpha_{0,1,2}(k)$

$$
\begin{aligned}
\alpha_{0}(k)= & \left(-\left.12 e^{+2 \xi-3 F} f\right|_{\partial \mathcal{B}}\right)\left(\int_{\mathcal{B}} d \xi e^{4 \xi-3 F}\right)^{-1} \\
\alpha_{1}(k)= & \left(\int_{\mathcal{B}} d \xi e^{+2 \xi-3 F}\left(-\phi_{1}-\frac{1}{8}\left(\frac{f^{\prime}}{2}+\bar{f} f\right)\right)\right) \\
& \times\left(\int_{\mathcal{B}} d \xi e^{4 \xi-3 F}\right)^{-1} \\
\alpha_{2}(k)= & \left(\int_{\mathcal{B}} d \xi e^{+2 \xi-3 F} \phi_{2}\right)\left(\int_{\mathcal{B}} d \xi e^{4 \xi-3 F}\right)^{-2} .
\end{aligned}
$$

As a reminder, the functions $\phi_{1,2}$ are explicitly given by

$$
\begin{aligned}
\phi_{1}(\xi)= & \frac{1}{2} \int_{-\frac{T_{k}}{2}}^{\xi} d \xi_{1}\left(e^{2\left(\xi-\xi_{1}\right)}-1\right) \mathbb{F}_{0}\left(\xi_{1}, k\right) \\
& -\frac{1}{2}\left(e^{2 \xi}-e^{\left.-T_{k}\right)} \int_{-\frac{T_{k}}{2}}^{\frac{T_{k}}{2}} d \xi_{1} e^{-2 \xi_{1}} \mathbb{F}_{0}\left(\xi_{1}, k\right)\right. \\
\phi_{2}(\xi)= & -\frac{1}{2} \int_{-\frac{T_{k}}{2}}^{\xi} d \xi_{1}\left(e^{2\left(\xi-\xi_{1}\right)}-1\right) e^{2 \xi_{1}-3 F\left(\xi_{1}, k\right)} \\
& +\frac{1}{2}\left(e^{2 \xi}-e^{-T_{k}}\right) \int_{-\frac{T_{k}}{2}}^{\frac{T_{k}}{2}} d \xi_{1} e^{-3 F\left(\xi_{1}, k\right)} .
\end{aligned}
$$

We fix the region of integration $\mathcal{B}$ to the period $T_{k}$ in (21) or $\mathcal{B}=\left[-\frac{T_{k}}{2}, \frac{T_{k}}{2}\right]$, with the boundary conditions specified earlier. The integrals in (62) for $\alpha_{1,2}(k)$ are not reducible to single integrals upon the insertion of (63) and integration by parts, since $e^{2 \xi-3 F}$ does not integrate to a simple function.

The first contribution in (60) is the holographic mass (25) of the tunneling configuration of order $\lambda N_{c}$, which is seen to reduce to the instanton mass (action) for $k=1$. The second contribution is the mass of the attached heavy quarks in the mesonic molecule. The contribution linear in $\lambda$ stems from the boundary term for the quadratic heavy meson action. It is nonzero for $0 \leq k<1$ due to the deviation of the tunneling gauge configuration from selfduality. The $\alpha_{1}$ contribution stems from the U(1) Coulomb coupling of the charge $\chi^{\dagger} \chi$ to the background charge $\rho_{0}$ and the Chern-Simons terms. The $\left(\chi^{\dagger} \chi\right)^{2}$ contribution stems from the U(1) Coulomb-like self-interaction. All of these contributions are similar to the $k=1$ case with the exception of the $\alpha_{0}$-contribution.

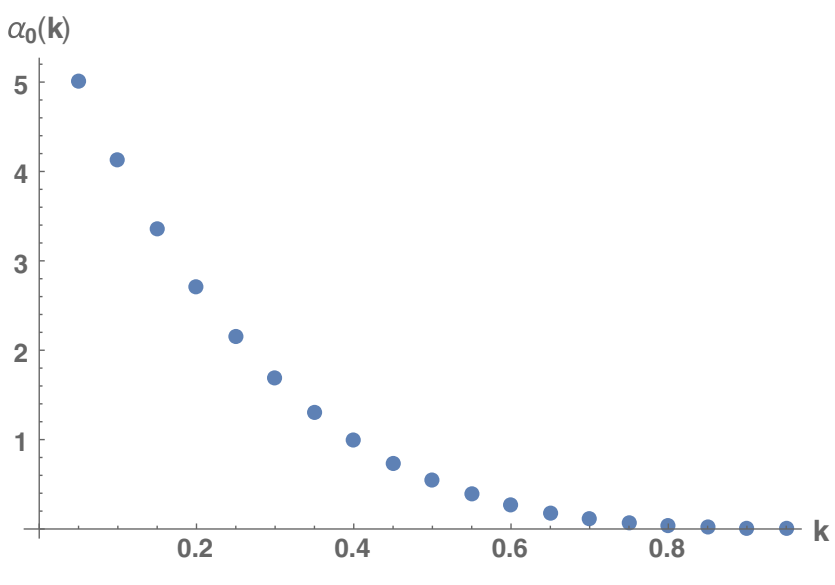

FIG. 5. $\alpha_{0}(k)$ vs $k$.

The behavior of $\alpha_{0,1,2}(k)$ vs $k$ is shown in Figs. 5-7, respectively. $\alpha_{0}(k)$ is maximally repulsive at $k=0$, reflecting on the maximal deviation from self-duality, and vanishes at the self-dual point with $k=1$, in agreement with Ref. [44]. $\alpha_{1}(k)$ is attractive for all $k$ with the expected value $\alpha_{1}(1)=-\frac{1}{8}$ from Ref. [44]. Equation $\alpha_{2}(k)$ is repulsive throughout with the limiting value $\alpha_{2}(1)=\frac{1}{3}$, in agreement with Ref. [44].

\section{Classical binding energy}

In general, the treatment of the size $\rho$ requires quantum mechanics as we detail below. However, for a classical estimate, we note that, since the dependence of $M_{k}$ vs $k$ is mild as seen in Fig. 3 with a size of order $\left(\lambda N_{c}\right)^{0} / \sqrt{\lambda}$, we may fix it near the instanton point at $k \sim 1$ with the result $[46,47]$

$$
\rho^{2}=\frac{27 \pi}{\lambda} \sqrt{\frac{6}{5}}
$$

in units of $M_{K K}$. If $N_{Q}=\chi_{Q}^{\dagger} \chi_{Q}$ is the number of heavy quarks (mesons) attached to the tunneling configuration,

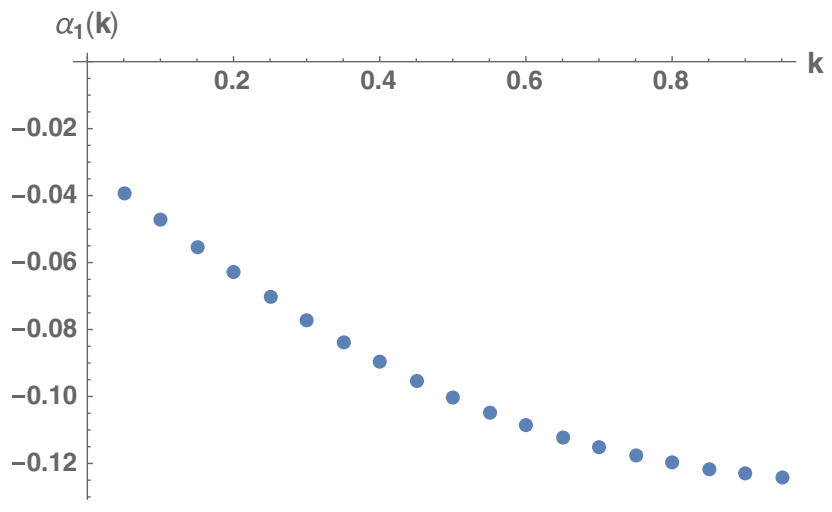

FIG. 6. $\alpha_{1}(k)$ vs $k$. 


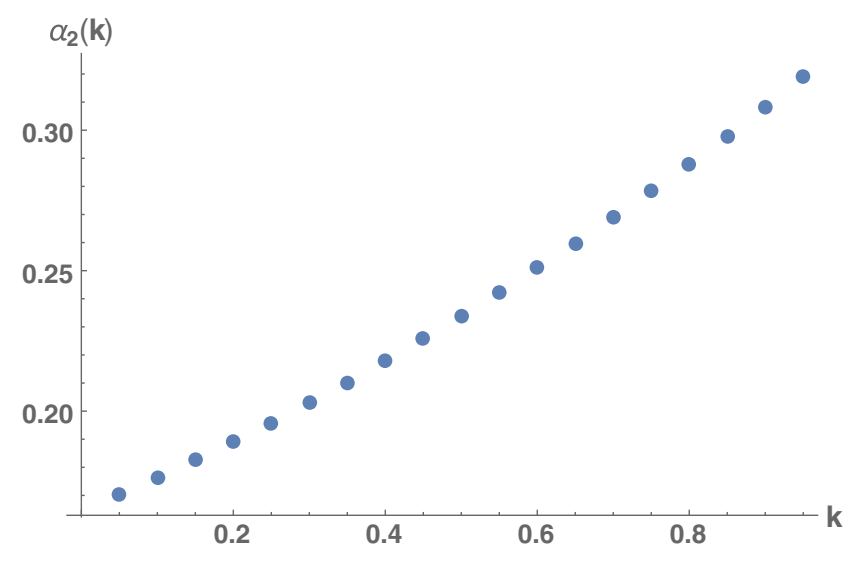

FIG. 7. $\alpha_{2}(k)$ vs $k$.

their classical binding energy $\Delta_{H}(k)$ as a function of $k$ follows from (60) as $\left(M_{1}=8 \pi^{2} \kappa\right)$

$$
\begin{aligned}
\Delta_{H}(k) \equiv & \left(\mathbb{H}_{k}-N_{Q} m_{H}\right) \\
\equiv & M_{k}+\left(\frac{\lambda \alpha_{0}(k)}{16 m_{H}}+162 \pi \alpha_{1}(k)\right) \frac{N_{Q}}{\rho^{2}} \\
& +27 \pi \alpha_{2}(k) \frac{N_{Q}^{2}}{N_{c} \rho^{2}} .
\end{aligned}
$$

We fix the holographic parameters to $N_{c}=3, \lambda=20$ [46,59] and $M_{K K}=m_{\rho} / \sqrt{0.67} \approx 1 \mathrm{GeV}$ [46]. For $N_{Q}=2$, we show in Fig. 8 the classical binding energy $\Delta_{H}(0)$ vs $m_{H}$ in units of $M_{K K}$. We recall that $k=0$ corresponds to the sphaleron path with zero topological charge. For charm and bottom, $m_{H}$ is fixed to the $\left(0^{-}, 1^{-}\right)$multiplet, i.e., $m_{D} \approx$ $1.870 \mathrm{GeV}$ for $\left(D, D^{*}\right)$ and $m_{B} \approx 5.279 \mathrm{GeV}$ for $\left(B, B^{*}\right)$. The classical binding of both charm and bottom is large and depends sensitively on the value of $\rho$. In the heavy quark limit with $m_{H} \rightarrow \infty$, the classical binding disappears when the repulsion $\left(\alpha_{2}\right)$ exceeds the attraction $\left(\alpha_{1}\right)$ modulo $M_{k}$, i.e., $N_{Q} / N_{c}>6\left|\alpha_{1}(0)\right| / \alpha_{2}(0) \approx 1.22$. A more accurate estimate of the binding requires a quantum treatment, as we now discuss.

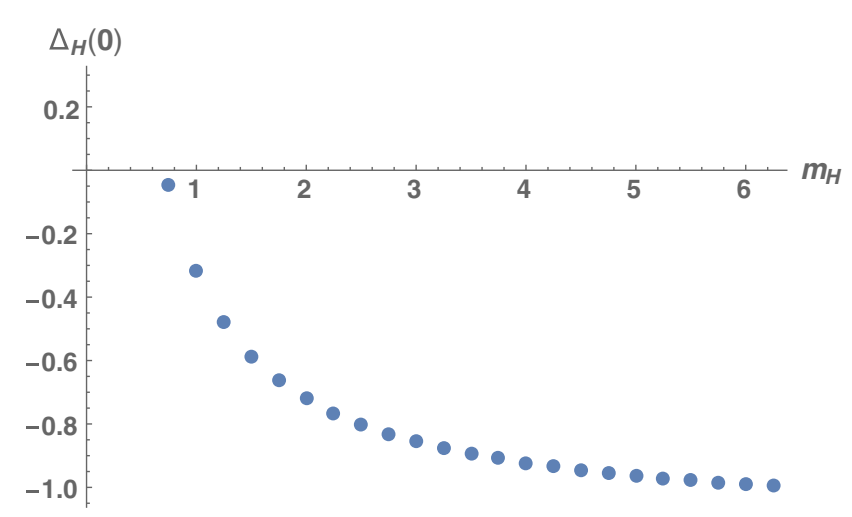

FIG. 8. Classical binding energy $\Delta_{H}(0)$ vs $m_{H}$ for $N_{Q}=2$ and $\lambda=20$ in units of $M_{K K}=1 \mathrm{GeV}$.

\section{E. Quantum moduli}

The quantum moduli space of the $\mathrm{O}(4)$ tunneling configuration is analogous to the instanton moduli or $R^{4} \times R^{4} / Z_{2}$ (flat space) [42]. Here, we focus on $R^{4} / Z_{2}$, which corresponds to the size and global flavor $\mathrm{SU}(2)$ orientations. We will refer to $y_{I}=\rho a_{I}$ as the coordinates on $R^{4} / Z_{2}$, with the $\mathrm{SU}(2)$ orientations parametrized by $a_{I}$ subject to the normalization $a_{I}^{2}=1$ and to $\rho$ as the size of the instanton. The collective Hamiltonian on the $R^{4} / Z_{2}$ moduli for the bound molecule follows from the arguments given in Ref. [44] as

$$
\begin{aligned}
\mathbf{H}= & -\frac{1}{2 m_{k}}\left(\frac{1}{\rho^{\frac{3}{2}}} \partial_{\rho}^{2} \rho^{\frac{3}{2}}+\frac{1}{\rho^{2}}\left(\nabla_{S^{3}}^{2}-2 m_{k} Q(k)\right)\right), \\
& +\frac{1}{2} m_{k} \omega_{k}^{2} \rho^{2}
\end{aligned}
$$

with $m_{k} / m_{1}=M_{k} / M_{1}$,

$$
\begin{aligned}
Q(k)= & \frac{N_{c}}{40 \pi^{2} a} \times\left(q(k)+\frac{\lambda}{m_{H}} \frac{5 \alpha_{0}(k)}{432 \pi} \frac{N_{Q}}{N_{c}}\right. \\
& \left.+30 \alpha_{1}(k) \frac{N_{Q}}{N_{c}}+5 \alpha_{2}(k) \frac{N_{Q}^{2}}{N_{c}^{2}}\right),
\end{aligned}
$$

and the inertial parameters $m_{1}=16 \pi^{2} a N_{c}, \omega_{1}^{2}=\frac{1}{6}$. Here, $q(k)$ is the $\mathrm{U}(1)$ topological self-repulsion in the absence of the heavy mesons

$$
q(k)=-\frac{45}{2} \int_{-\frac{T_{k}}{2}}^{\frac{T_{k}}{2}} e^{-2 \xi} \phi_{1}(\xi) \mathbb{F}_{0}[\xi, k] .
$$

In Fig. 9, we show (68) vs $k$, which is seen to increase monotonously with the topological charge, from $q(0)=0$ on the sphaleron path to $q(1)=1$ on the instanton path [44].

All contributions in (67) are in principle leading in the triple limit $N_{c}>\lambda>m_{H} \gg 1$, provided that $N_{Q} / N_{c}$ is

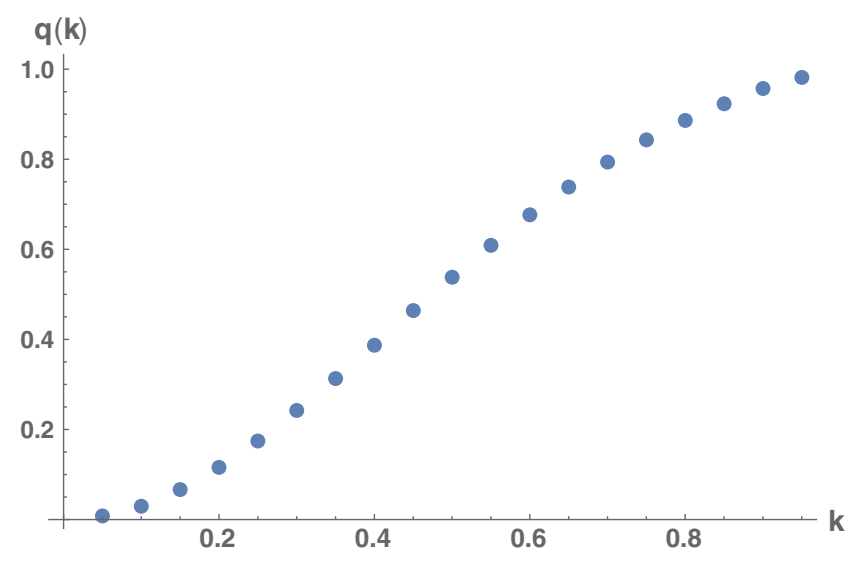

FIG. 9. U(1) self-repulsion $q(k)$ vs $k$. 
of order 1. However, in practice, some of the inequalities may not be fulfilled. This is a known shortcoming of the holographic construction, where for instance $\lambda>N_{c}$ is used in most applications [42,46,59].

For $N_{Q}=0$, the eigenstates of (66) are given by $T_{l}(a) R_{l n}$, where $T_{l}(a)$ are the spherical harmonics on $S^{3}$ with $\nabla^{2} T_{l}=-l(l+2) T_{l}$. Under $\mathrm{SO}(4) \sim \mathrm{SU}(2) \times \mathrm{SU}(2)$, they are in the $\left(\frac{l}{2}, \frac{l}{2}\right)$ representations, with the two $S U(2)$ identified by the isometry $a_{I} \rightarrow V_{L} a_{I} V_{R}$. The left factor is the isospin rotation, and the right factor is the space rotation with quantum numbers $I=J=\frac{l}{2}$.

For $N_{Q} \neq 0$, the isospin (I) and the spin (J) decouple with the identification [44]

$$
\mathbf{J}=-\mathbf{I}+\chi_{Q}^{\dagger} \mathbf{T} \chi_{Q}
$$

The isospin-spin representations are now

$$
I J \equiv\left(\frac{l}{2}, \frac{l}{2}\right) \rightarrow\left(\frac{l}{2}, \frac{l}{2} \bigoplus_{i=1}^{N_{Q}} \frac{1}{2}\right)
$$

\section{F. Multiquark exotics}

The radial equation for the reduced wave function $R_{n l}=u_{n l} / \rho^{\frac{3}{2}}$ following from (66) now reads

$$
-u_{n l}^{\prime \prime}+\frac{g_{l}(k)}{\rho^{2}} u_{n l}+\left(m_{k} \omega_{k} \rho\right)^{2} u_{n l}=e_{k, n l} u_{n l}
$$

with

$$
g_{l}(k)=l(l+2)+2 m_{k} Q(k)
$$

and $e_{k, n l}=2 m_{k}\left(E_{k, n l}-M_{k}-N_{Q} m_{H}\right)$. The quantum corrected classical binding energy (65) is now

$$
\Delta_{H}(k) \rightarrow \Delta_{k}=E_{k, n l}-N_{Q} m_{H}=M_{k}+\frac{e_{k, n l}}{2 m_{k}} .
$$

The occurrence of the $1 / \rho^{2}$ potential at short distances is a key feature of the holographic formulation. It stems from the nature of the attraction in (60), which is dipolelike, and the repulsion in (60), which is Coulombic in four spatial dimensions. (Recall that Coulomb law is $1 / \rho$ in three spatial dimensions). It is dominant at small distances, with the critical coupling of $-\frac{1}{4}$ for the formation of deep bound states below the heavy meson threshold. Throughout, binding means that $E_{k, n l}-N_{Q} m_{H}<0$.

In Fig. 10, we show the behavior of $s_{l}(x) \equiv g_{l}(0)+\frac{1}{4}$ as a function of $x=N_{Q} / N_{c}$ with $m_{H} \rightarrow \infty$, on the sphaleron path (zero topological charge), for the $l=0$ lower (blue) curve, $l=1$ middle (orange) curve, and $l=2$ upper (green) curve. Only for $l=0$ and $x=N_{Q} / N_{c}<1.2$ is the attraction sufficiently strong to form deep bound states.

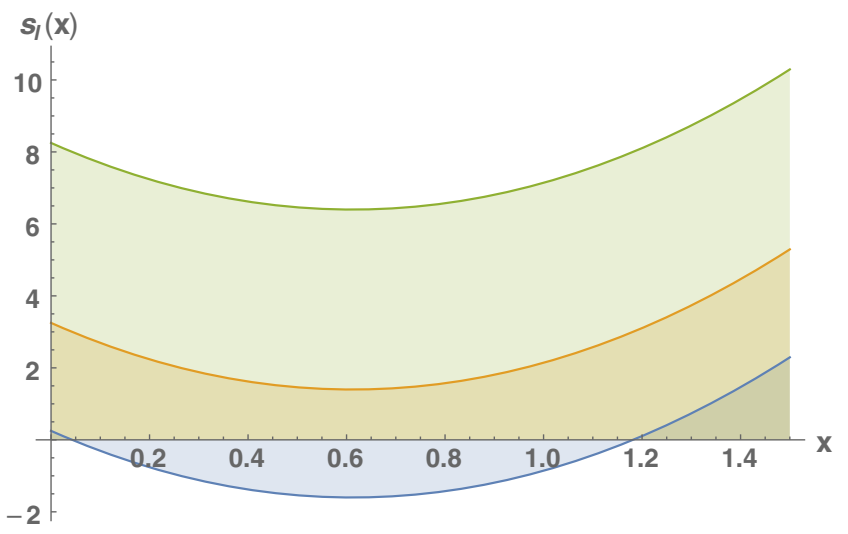

FIG. 10. $s_{l}(x) \equiv g_{l}(0)+\frac{1}{4}$ vs $x=N_{Q} / N_{c}$ for the $l=0$ lower (blue) curve, $l=1$ middle (orange) curve, and $l=2$ upper (green) curve.

Higher waves with $l=1,2, \ldots$ are unbound. For $N_{c}=3$, only $N_{Q} \leq 3$ states are a priori bound, i.e., open-flavor tetraquark $Q Q \bar{q} \bar{q}$ and hexaquark $Q Q Q \bar{q} \bar{q} \bar{q}$ states. The S-wave tetraquark states $Q Q \bar{q} \bar{q}$ carry $I J^{\pi}=00^{+}, 01^{+}$ assignments with Chern-Simons number $+\frac{1}{2}$ and are degenerate.

\section{G. Efimov states}

For small distances and S-waves, Eq. (71) reduces to

$$
-u_{n 0}^{\prime \prime}+\frac{g_{0}(k)}{\rho^{2}} u_{n 0} \approx e_{k, n 0} u_{n 0}
$$

For $g_{0}(k)+\frac{1}{4}<0$, the potential in (74) is sufficiently attractive to form deep bound states. However, it is singular and requires regularization [60]. The scale invariance of (74) allows for a universal regularization using the renormalization group approach, whereby the depth of the attractive and singular potential can be chosen to be a function of a short distance cutoff $R_{S}$ [60]. As a result, Eq. (74) admits many S-wave bound states with an accumulation near threshold, the so-called Efimov states [61]. We note that in our case there is a minimal value for the cutoff $R_{\min }=1 / m_{H}$, so the number of bound states is limited.

The bound state spectrum for (74) was detailed in Ref. [60] with extensive analysis in the context of the renormalization group cycle, with the result

$$
\begin{aligned}
e_{k, n 0} & =-\frac{4}{r_{0}^{2}} e^{\varphi_{k, n}} \\
\varphi_{k, n} & =\left(\frac{2}{\nu_{k, x}}\left(C+\operatorname{Im} \ln \Gamma\left(1+i \nu_{k, x}\right)-\left(n+\frac{1}{2}\right) \pi\right)\right) \\
\nu_{k, x} & =\left(-\frac{1}{4}-g_{0}(k)\right)^{\frac{1}{2}}
\end{aligned}
$$




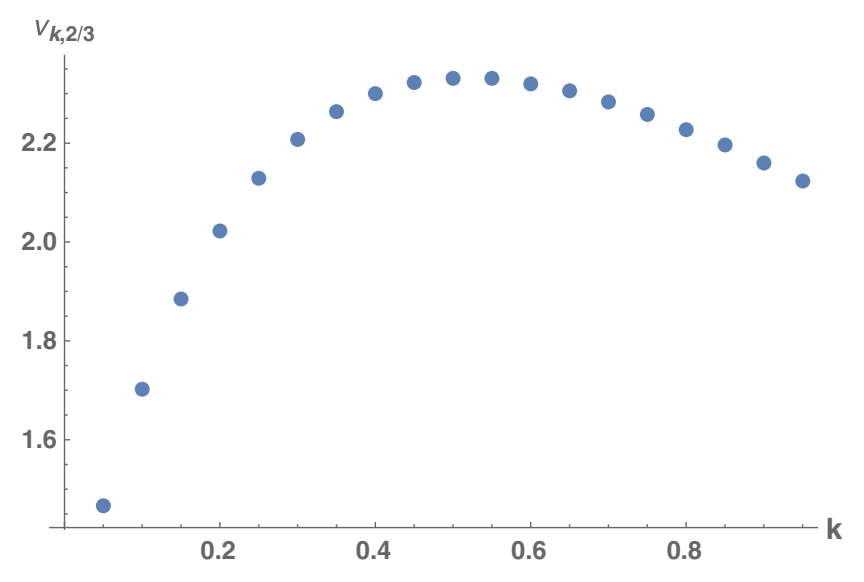

FIG. 11. $\nu_{k, 2 / 3}$ vs $k$ for $x=\frac{2}{3}$ and $m_{H} \rightarrow \infty$.

where $x=N_{Q} / N_{c}$. To avoid cluttering the notations, we omitted the x-dependence in all quantities except $\nu_{k, x}$. The behavior of $\nu_{k, x}$ vs $k$ is shown in Fig. 11 for $N_{c}=3$, $x=2 / 3$ and $m_{H} \rightarrow \infty$. The coupling peaks at $k=\frac{1}{2}$, halfway between the instanton and sphaleron path. Consecutive bound state energies are tied geometrically,

$$
\frac{e_{k,(n+1) 0}}{e_{k, n 0}}=e^{-\frac{2 \pi}{\nu_{k, x}}}
$$

showing their accumulation or dissipation at threshold $e_{k, 00}=0$, i.e., the Efimov effect. The undetermined constant $C$ in the quantum spectrum (75) reflects on the singular potential, that warrants renormalization. Changing $C$ amounts to redefining the depth of the singular potential.

In (75), the scale $r_{0}$ is fixed by the curvature or range of the long distance $1 / \rho^{2}$ potential. From (71), we fix it by matching the strength of the short distance potential to the strength of the large distance harmonic potential, i.e., $r_{0}^{2}=1 /\left(m_{k} \omega_{1}\right)$. As a result, the quantum binding energy (73) becomes

$$
\begin{aligned}
\Delta_{k}\left(N_{c}, x, \lambda\right) & =M_{k}-2 \omega_{1} e^{\varphi_{k, n}} \\
& =\left(\frac{\lambda N_{c}}{27 \pi}\right) \frac{M_{k}}{M_{1}}-\sqrt{\frac{2}{3}} e^{\varphi_{k, n}} .
\end{aligned}
$$

For $N_{c}=3$ and at the sphaleron point with $k=0$, the binding energy (77) depends on the coupling $\lambda$, the occupation ratio $x=N_{Q} / N_{c}<1.2$, and the parameter $C$ (cutoff depth). We fix $C$ so that the binding energy vanishes for $x=1 / 3$ giving a $Q \bar{q}$-meson state of energy exactly $m_{H}$. With this in mind, Eq. (77) reads

$$
\begin{aligned}
\Delta_{0}(3, \lambda, x)= & \frac{\lambda}{24 \sqrt{2}}-\sqrt{\frac{2}{3}}\left(\frac{\lambda \sqrt{3}}{48}\right)^{\nu_{0,1 / 3} / \nu_{0, x}} \\
& \times e^{\frac{2}{\nu_{0, x}}\left(\operatorname{Im} \operatorname{Ln} \Gamma\left(1+i \nu_{0, x}\right)-\operatorname{Im} \operatorname{Ln} \Gamma\left(1+i \nu_{0,1 / 3}\right)\right) .}
\end{aligned}
$$

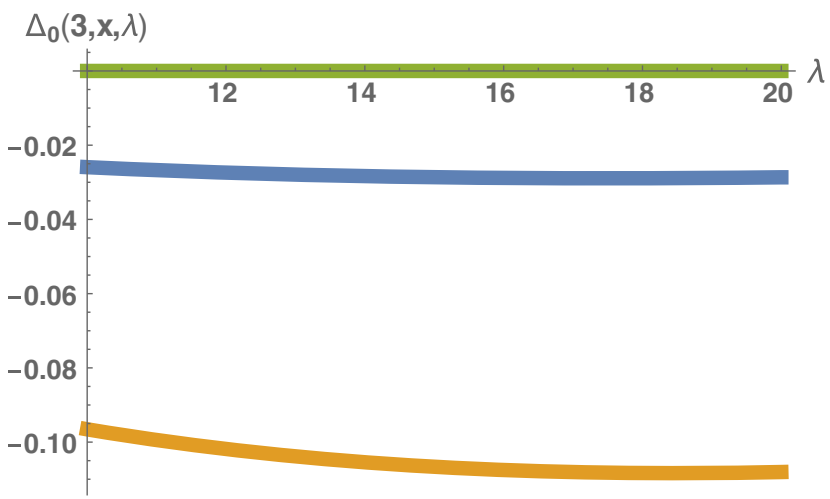

FIG. 12. Binding energy (78) vs $\lambda$ for $m_{H} \rightarrow \infty: x=\frac{1}{3}$ green (upper) curve, $x=\frac{2}{3}$ lower (orange) curve, and $x=\frac{3}{3}$ middle (blue) curve.

In the heavy quark limit, $\nu_{0,1 / 3}=1.10, \nu_{0,2 / 3}=1.26$, and $\nu_{0,3 / 3}=0.93$.

Figure 12 shows the behavior of the binding energies (78) vs the 't Hooft coupling $\lambda$, for the $x=\frac{1}{3}$ upper (green) curve, $x=\frac{2}{3}$ lower (orange) curve, and $x=\frac{3}{3}$ middle (blue) curve, in the heavy quark limit. For $m_{H} \rightarrow \infty$, the binding energies of the tetraquark states $Q Q \bar{q} \bar{q}$ and hexaquark states $Q Q Q \bar{q} \bar{q} \bar{q}$ are listed in the first columns of Tables I and II, respectively. The binding energies are not very sensitive to $10 \leq \lambda \leq 20$, which is the range favored by the light meson and baryon dynamics [42,46]. For bottom B-mesons with $m_{B}=5.279 \mathrm{GeV}$ and charmed D-mesons with $m_{D}=1.87 \mathrm{GeV}$, the binding energies for tetraquark and hexaquark states are also listed in Tables I and II, respectively. The hexaquark states are unbound for finite masses. The results do not change sensitively if we were to use the $\mathrm{B}^{*}$-meson and $\mathrm{D}^{*}$-meson masses instead.

We note that for the bound tetraquark states the Efimov factor is $e^{-2 \pi / \nu_{0,2 / 3}} \approx 10^{-3}$. This factor in the geometrical ratio (76) shows that the radially excited tetraquark exotics rapidly move to the continuum and unbind. So, we expect only one bound state to survive.

The heavy multiquark states with hidden flavor are also covered by the present analysis provided that $N_{Q} \rightarrow N_{Q}-N_{\bar{Q}}$ is substituted in (67) when $N_{Q}>N_{\bar{Q}}$. For $N_{Q}<N_{\bar{Q}}$, the starting $\mathrm{O}(4)$ tunneling configuration should be an anti-instanton path with topological charge -1 , interpolating to an antisphaleron path with ChernSimons number $-\frac{1}{2}$. This implies conjugate symmetry for bosonic multiquark states. As a result, the conjugate

TABLE I. Binding energies for tetraquarks.

\begin{tabular}{lcccc}
\hline \hline$\lambda$ & $Q Q \bar{q} \bar{q}(\mathrm{GeV})$ & $b b \bar{q} \bar{q}(\mathrm{GeV})$ & $b c \bar{q} \bar{q}(\mathrm{GeV})$ & $c c \bar{q} \bar{q}(\mathrm{GeV})$ \\
\hline 10 & -0.097 & -0.088 & -0.080 & -0.072 \\
15 & -0.107 & -0.091 & -0.077 & -0.062 \\
20 & -0.108 & -0.085 & -0.064 & -0.041 \\
\hline \hline
\end{tabular}


TABLE II. Binding energies for hexaquarks.

\begin{tabular}{lcccc}
\hline \hline & $\begin{array}{c}Q Q Q \bar{q} \bar{q} \bar{q} \\
(\mathrm{GeV})\end{array}$ & $\begin{array}{c}b b b \bar{q} \bar{q} \bar{q} \\
(\mathrm{GeV})\end{array}$ & $\begin{array}{c}b b c \bar{q} \bar{q} \bar{q} \\
(\mathrm{GeV})\end{array}$ & $\begin{array}{c}c c c \bar{q} \bar{q} \bar{q} \\
(\mathrm{GeV})\end{array}$ \\
\hline 10 & -0.026 & -0.005 & +0.008 & +0.037 \\
15 & -0.029 & +0.006 & +0.029 & +0.076 \\
20 & -0.029 & +0.018 & +0.045 & +0.084 \\
\hline \hline
\end{tabular}

tetraquark states $\bar{Q} \bar{Q} q q$ are bound and degenerate with $Q Q \bar{q} \bar{q}$. Heavier tetraquark states such as $Q Q \bar{Q} \bar{Q}$ with $N_{Q} \rightarrow N_{Q}-N_{\bar{Q}}=0$ are unbound. Heavier multiquark states of the type $Q Q \bar{Q} \bar{q}$ and $Q Q \bar{Q} q \bar{q} \bar{q}$ with $N_{Q} \rightarrow N_{Q}-$ $N_{\bar{Q}}=1$ are unbound, but those of the type $Q Q(Q \bar{Q})^{p} \bar{q} \bar{q}$ with $N_{Q}-N_{\bar{Q}}=(p+2)-p=2$ are bound in the heavy quark limit.

The holographic exotics with hidden flavor $Q \bar{Q} q \bar{q}$ with $N_{Q} \rightarrow N_{Q}-N_{\bar{Q}}=0$ are also unbound. Experimental evidence in Refs. [1-4] suggests otherwise. This shows that the X,Y,Z states reported in Refs. [1-4] are not the compact topological molecules discussed here but likely loosely bound hadronic molecules (deusons) [7,8,10-15]. Finally, we also note that the present analysis is limited to the light $\mathrm{SU}(2)$ flavor sector. The extension to the SU(3) flavor sector with massive strange quarks is more involved [44] and will be discussed elsewhere.

\section{H. Discussion}

Recent lattice and phenomenological estimates suggest that the double-bottom tetraquark state is deeply bound with $\Delta_{B B}=-(0.15-0.2) \mathrm{GeV}$ [62] (lattice) and $\Delta_{B B}=$ $-(0.17) \mathrm{GeV}$ [23]. The same lattice analysis suggests that the mixed charm-bottom tetraquark state is bound $\Delta_{C B}=-(0.061-0.015) \mathrm{GeV}$, but the double-charm tetraquark state is not $[62,63]$.

In the coupling range $10 \leq \lambda \leq 20$ suggested by the holographic analysis of the light hadrons $[42,46]$, our results support a double-bottom tetraquark state with a binding energy $\Delta_{B B}=-(0.088-0.091) \mathrm{GeV}$ somewhat lower than the lattice estimate, a mixed bottom-charm tetraquark with a binding energy $\Delta_{C B}=-(0.064-0.080) \mathrm{GeV}$ closer to the lattice estimate. They support a bound double-charm tetraquark with a binding energy $\Delta_{C C}=-(0.041-0.072) \mathrm{GeV}$ contrary to the lattice estimate, although larger values of $\lambda$ may cause it to unbind. These results are overall consistent with earlier estimates in the context of the random instanton model [22].

Finally, we note that multiquark exotics in the context of holography have been recently addressed in the context of the holography inspired stringy hadron model (HISH) [55]. We view our analysis as complementary to the HISH analysis as it applies to the low lying exotics as opposed to the highly excited and stringy exotics. Our analysis can be extended to the excited and unbound exotic states for slow rotations, with the rotational-vibrational spectrum for $l>0$,

$$
E_{0, n l}=M_{0}+N_{Q} m_{H}+\left(\frac{l^{2}}{6}+\frac{2 N_{c}}{81} Q(0)\right)^{\frac{1}{2}}+\frac{2 n+1}{\sqrt{6}},
$$

following from (66)-(67) through standard arguments $[42,44]$. The states described by (70) are unstable against the strong decay to heavy-light mesons. For $l \gg 1$, the spectrum $(79)$ does not Reggeize since $E_{0, n l} \approx l$. A way to achieve Reggeization is through relativistic rotations, that allow for a stringylike deformation of the underlying $\mathrm{O}(4)$ tunneling configuration. This will be discussed elsewhere.

\section{CONCLUSIONS}

We have presented a top-down holographic approach to multiquark exotic states using a minimally modified D4-D8-D $\overline{8}$ setup to account for two light and one heavy flavors [44]. The heavy multiquark states are topological molecules of heavy-light mesons bound to a tunneling gauge configuration with fixed Chern-Simons number. The latter interpolates between an instanton path with net topological charge 1 or baryon number (a fermion) and a sphaleron path with net topological charge 0 and Chern-Simons number $\frac{1}{2}$ (a boson).

The geometrical interpolation between a fermion and a boson in higher dimensions is remarkable. It points to a topological duality between the heavy baryon exotics discussed in Ref. [44] and the heavy meson exotics addressed here. This is perhaps suggestive of a geometrical realization and generalization of the Savage-Wise symmetry [64] to most heavy exotics.

In leading order in the heavy quark limit, the bounded heavy mesons to the tunneling path with fixed ChernSimons number transmute to fermions. This mechanism is reminiscent of the transmutation of the strange quark spin to the Skyrmion in the kaon-Skyrmion bound state [65]. The binding of the fermions follows by balancing the attraction induced by the Chern-Simons term which is dipolelike and the dual repulsion stemming from the induced $U(1)$ gauge field together with the deviation of the tunneling configuration from self-duality. All these contributions are four-dimensional Coulomb like and scale like $1 / \rho^{2}$ (instead of $1 / \rho$ for three-dimensional Coulomb). The ensuing potential in the molecule is singular. As a result, the topologically bound exotics are Efimov states.

Our analysis shows that only the open-flavor molecules with $x=N_{Q} / N_{c}<1.2$ are bound in the heavy quark limit. For $N_{c}=3$, the open-flavor exotics $Q Q \bar{q} \bar{q}$ and their conjugate $\bar{Q} \bar{Q} q q$ are bound in a degenerate multiplet $I J^{\pi}=\left(00^{+}, 01^{+}\right)$with opposite intrinsic Chern-Simons numbers $\pm \frac{1}{2}$. The open-flavor and nonstrange hexaquark states $Q Q Q \bar{q} \bar{q} \bar{q}$ are bound in the heavy quark limit only. 
The heavier exotics $Q Q \bar{Q} \bar{Q}$ are unbound. The compact exotics with hidden flavor such as $Q Q \bar{Q} \bar{q}$ and $Q \bar{Q} q \bar{q}$ are also unbound, but the heavier exotics such as $Q Q(Q \bar{Q}) \bar{q} \bar{q}$ are bound in the heavy quark limit.

The leading holographic correction in the heavy quark mass is found to penalize the binding in $c c \bar{q} \bar{q}$ more than in $b b \bar{q} \bar{q}$. Our analysis suggests a rotational-vibrational tower of multiquark excitations prone to strong decay.

Some of the shortcomings of the present approach lie in the use of the triple limits of large $N_{c}$, large coupling $\lambda$, and large meson mass $m_{H}$, with $N_{Q} / N_{c}$ of order 1 . Although the relaxation of these limits is straightforward in principle, its systematic implementation is involved in practice. This not withstanding, the present setup is noticeable because of the limited number of parameters it carries. The brane tension $\kappa \sim \lambda N_{c}$ is usually traded for the pion decay constant and the Kaluza-Klein scale for the rho meson mass all in the light meson sector, leaving the treatment of the heavy-light sector parameter free modulo the heavy meson masses $m_{H}$.

Unlike most of the approaches for heavy exotics (see Ref. [66] and references therein), the present construction enforces heavy quark symmetry and the spontaneous breaking of chiral symmetry; provides a systematic organizational framework using the QCD parameters $N_{c}, \lambda, m_{H}$; and solves the multibody problem using topological bound states.

The construction can be improved in a number of ways, for instance, by breaking isospin symmetry and including strangeness to account for strange topological exotics or by adding a tachyon and a tachyon potential to bring the model closer to QCD at short distances, perhaps in the context of improved holographic QCD [67]. These and related issues will be discussed next.

\section{ACKNOWLEDGMENTS}

We thank Marek Karliner for a discussion. M. A. N. thanks the Nuclear Theory Group at Stony Brook University for hospitality during the completion of this work. This work was supported by the U.S. Department of Energy under Contract No. DE-FG-88ER40388 and by the Polish National Science Centre (NCN) Grant No. UMO2017/27/B/ST2/01139.

\section{APPENDIX A: HEAVY-LIGHT ACTION}

The explicit construction of the holographic heavy-light action was detailed in Ref. [44]. Here, we quote the relevant expressions for (11) for completeness,

$$
\begin{aligned}
\mathcal{L}_{0}= & -\left(D_{M} \Phi_{N}^{\dagger}-D_{N} \Phi_{M}^{\dagger}\right)\left(D_{M} \Phi_{N}-D_{N} \Phi_{M}\right)+2 \Phi_{M}^{\dagger} F_{M N} \Phi_{N} \\
\mathcal{L}_{1}= & +2\left(D_{0} \Phi_{M}^{\dagger}-D_{M} \Phi_{0}^{\dagger}\right)\left(D_{0} \Phi_{M}-D_{M} \Phi_{0}\right)-2 \Phi_{0}^{\dagger} F^{0 M} \Phi_{M}-2 \Phi_{M}^{\dagger} F^{M 0} \Phi_{0}-2 m_{H}^{2} \Phi_{M}^{\dagger} \Phi_{M}+\tilde{\mathcal{L}}_{1} \\
\mathcal{L}_{C S}= & -\frac{i N_{c}}{24 \pi^{2}}\left(d \Phi^{\dagger} A d \Phi+d \Phi^{\dagger} d A \Phi+\Phi^{\dagger} d A d \Phi\right)-\frac{i N_{c}}{16 \pi^{2}}\left(d \Phi^{\dagger} A^{2} \Phi+\Phi^{\dagger} A^{2} d \Phi+\Phi^{\dagger}(A d A+d A A) \Phi\right) \\
& -\frac{5 i N_{c}}{48 \pi^{2}} \Phi^{\dagger} A^{3} \Phi+S_{C}\left(\Phi^{4}, A\right)
\end{aligned}
$$

and

$$
\begin{aligned}
\tilde{\mathcal{L}}_{1}= & +\frac{1}{3} z^{2}\left(D_{i} \Phi_{j}-D_{j} \Phi_{i}\right)^{\dagger}\left(D_{i} \Phi_{j}-D_{j} \Phi_{i}\right) \\
& -2 z^{2}\left(D_{i} \Phi_{z}-D_{z} \Phi_{i}\right)^{\dagger}\left(D_{i} \Phi_{z}-D_{z} \Phi_{i}\right) \\
& -\frac{2}{3} z^{2} \Phi_{i}^{\dagger} F_{i j} \Phi_{j}+2 z^{2}\left(\Phi_{z}^{\dagger} F_{z i} \Phi_{i}+\text { c.c. }\right) .
\end{aligned}
$$

\section{APPENDIX B: O(3) SYMMETRIC TUNNELING SOLUTION AND ITS FERMIONIC ZERO MODE}

In this Appendix, we suggest yet another tunneling configuration with $\mathrm{O}(3)$ instead of $\mathrm{O}(4)$ symmetry that is also suitable for binding heavy-light mesons. This tunneling configuration is fully localized in flat $R^{4}$. This configuration is characterized by a turning point in the holographic direction at $z=0$, in agreement with the explosive sphaleron configurations discussed in Refs. [56-58]. To construct it, we note that the $\mathrm{O}(4)$ solutions to the Yang-Mills equations with a turning point at $\xi=0$ relate to the solution with a turning point at $z=0$ by the inversion

$$
(x+a)_{M}=\frac{2 \rho^{2}}{|y+a|^{2}}(y+a)_{M}
$$

with $a=(\overrightarrow{0}, \rho)$, which maps the sphere $y^{2}=\rho^{2}$ onto the upper-half of the $\mathrm{x}$ space as illustrated in Fig. 13. This

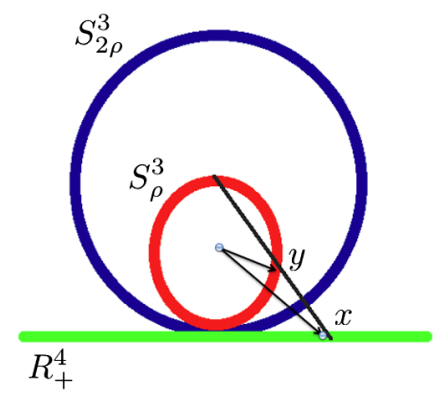

FIG. 13. Inversion of $S_{\rho}^{3}$ onto $R_{+}^{4}$ through $S_{2 \rho}^{3}$. 
inversion leaves the line element in $R^{4}$ unchanged modulo a conformal weight $\sigma(y)$,

$$
|d y|^{2}=\sigma(y)|d x|^{2}=\frac{|y+a|^{4}}{4 \rho^{4}}|d x|^{2},
$$

and leaves invariant the 1 -form of the gauge field

$$
d x_{\mu} A_{\mu}(x)=d y_{\nu} A_{\nu}(y)
$$

This leads to the transform

$A_{a M}(x)=\sqrt{\sigma(y)}\left(g_{M N}-2 \frac{(y+a)_{M}(y+a)_{N}}{(y+a)^{2}}\right) A_{a N}(y)$

with $y$ solving (B1).

We now proceed to construct the $\mathrm{O}(3)$ symmetric zero mode by applying the spatial inversion (B1) onto the $\mathrm{O}(4)$ symmetric zero mode in (46) through

$$
\tilde{\psi}_{+}(x)=\frac{\sigma_{\mu}^{\dagger}(y+a)_{\mu}}{1 /(y+a)^{2}} \psi_{+}(y)
$$

More explicitly, we have $(r=|\vec{x}|)$

$$
\begin{aligned}
\tilde{\psi}_{+}(r, z)= & \frac{8 \rho^{6} \mathbf{C}}{\left((z+\rho)^{2}+r^{2}\right)^{2}} \\
& \times((z+\rho)+i \vec{\sigma} \cdot \vec{x}) e^{-\frac{3}{2} F(\xi(y))} \chi_{Q}
\end{aligned}
$$

with

$$
\xi(y)=\frac{1}{2} \ln \left(\frac{(z-\rho)^{2}+r^{2}}{(z+\rho)^{2}+r^{2}}\right) .
$$

This result is in agreement with the one derived in Ref. [58] prior to the analytical continuation to Minkowski space [see their Eq. (22) with a minor correction of the 2 to $\frac{3}{2}$ in their exponent].
[1] I. Adachi (Belle Collaboration), arXiv:1105.4583; A. Bondar et al. (Belle Collaboration), Phys. Rev. Lett. 108, 122001 (2012).

[2] M. Ablikim et al. (BESIII Collaboration), Phys. Rev. Lett. 110, 252001 (2013).

[3] V. M. Abazov et al. (D0 Collaboration), Phys. Rev. Lett. 117, 022003 (2016).

[4] R. Aaij et al. (LHCb Collaboration), Phys. Rev. Lett. 118, 022003 (2017); Phys. Rev. D 95, 012002 (2017).

[5] R. Aaij et al. (LHCb Collaboration), Phys. Rev. Lett. 115, 072001 (2015); 117, 082002 (2016); 117, 082003 (2016); 117, 109902(A) (2016).

[6] R. Aaij et al. (LHCb Collaboration), Phys. Rev. Lett. 118, 182001 (2017).

[7] M. B. Voloshin and L. B. Okun, Pis'ma Zh. Eksp. Teor. Fiz. 23, 369 (1976) [JETP Lett. 23, 333 (1976)].

[8] N. A. Tornqvist, Phys. Rev. Lett. 67, 556 (1991); Z. Phys. C 61, 525 (1994); Phys. Lett. B 590, 209 (2004).

[9] L. Maiani, F. Piccinini, A. D. Polosa, and V. Riquer, Phys. Rev. D 71, 014028 (2005); S. Dubynskiy and M. B. Voloshin, Phys. Lett. B 666, 344 (2008); L. Maiani, V. Riquer, R. Faccini, F. Piccinini, A. Pilloni, and A. D. Polosa, Phys. Rev. D 87, 111102 (2013); L. Maiani, F. Piccinini, A. D. Polosa, and V. Riquer, Phys. Rev. D 89, 114010 (2014); A. Pilloni C. Fernández-Ramírez, A. Jackura, V. Mathieu, M. Mikhasenko, J. Nys, and A. P. Szczepaniak (JPAC Collaboration), Phys. Lett. B 772, 200 (2017).

[10] M. Karliner and H. J. Lipkin, arXiv:0802.0649; M. Karliner and J. L. Rosner, Phys. Rev. Lett. 115, 122001 (2015); M. Karliner, Acta Phys. Pol. B 47, 117 (2016).
[11] C. E. Thomas and F.E. Close, Phys. Rev. D 78, 034007 (2008); F. Close, C. Downum, and C. E. Thomas, Phys. Rev. D 81, 074033 (2010).

[12] S. Ohkoda, Y. Yamaguchi, S. Yasui, K. Sudoh, and A. Hosaka, Phys. Rev. D 86, 034019 (2012); Few-Body Syst. 54, 1019 (2013).

[13] M. T. AlFiky, F. Gabbiani, and A. A. Petrov, Phys. Lett. B 640, 238 (2006); I. W. Lee, A. Faessler, T. Gutsche, and V. E. Lyubovitskij, Phys. Rev. D 80, 094005 (2009); M. Suzuki, Phys. Rev. D 72, 114013 (2005); J. R. Zhang, M. Zhong, and M. Q. Huang, Phys. Lett. B 704, 312 (2011); D. V. Bugg, Europhys. Lett. 96, 11002 (2011); J. Nieves and M. P. Valderrama, Phys. Rev. D 84, 056015 (2011); M. Cleven, F. K. Guo, C. Hanhart, and U. G. Meissner, Eur. Phys. J. A 47, 120 (2011); T. Mehen and J. W. Powell, Phys. Rev. D 84, 114013 (2011); F. K. Guo, C. Hidalgo-Duque, J. Nieves, and M. P. Valderrama, Phys. Rev. D 88, 054007 (2013); Q. Wang, C. Hanhart, and Q. Zhao, Phys. Rev. Lett. 111, 132003 (2013); F. K. Guo, C. Hanhart, Q. Wang, and Q. Zhao, Phys. Rev. D 91, 051504 (2015); X. W. Kang, Z. H. Guo, and J. A. Oller, Phys. Rev. D 94, 014012 (2016); X. W. Kang and J. A. Oller, Eur. Phys. J. C 77, 399 (2017).

[14] E. S. Swanson, Phys. Rep. 429, 243 (2006); Z. F. Sun, J. He, X. Liu, Z. G. Luo, and S. L. Zhu, Phys. Rev. D 84, 054002 (2011).

[15] Y. Liu and I. Zahed, Phys. Lett. B 762, 362 (2016); Y. Liu and I. Zahed, Int. J. Mod. Phys. E 26, 1740017 (2017); Phys. Lett. B 769, 314 (2017).

[16] A. V. Manohar and M. B. Wise, Nucl. Phys. B399, 17 (1993); N. Brambilla et al., Eur. Phys. J. C 71, 1534 (2011); 
M. B. Voloshin, Prog. Part. Nucl. Phys. 61, 455 (2008); J. M. Richard, Few-Body Syst. 57, 1185 (2016).

[17] D. O. Riska and N. N. Scoccola, Phys. Lett. B 299, 338 (1993).

[18] T. F. Carames, A. Valcarce, and J. Vijande, Phys. Lett. B 699, 291 (2011); J. M. Richard, A. Valcarce, and J. Vijande, Phys. Rev. C 97, 035211 (2018); A. Ali, Q. Qin, and W. Wang, Phys. Lett. B 785, 605 (2018).

[19] M. Nielsen, F. S. Navarra, and S. H. Lee, Phys. Rep. 497, 41 (2010).

[20] J. P. Ader, J. M. Richard, and P. Taxil, Phys. Rev. D 25, 2370 (1982); S. Zouzou, B. Silvestre-Brac, C. Gignoux, and J. M. Richard, Z. Phys. C 30, 457 (1986); J. Carlson, L. Heller, and J. A. Tjon, Phys. Rev. D 37, 744 (1988).

[21] M. A. Nowak, I. Zahed, and M. Rho, Phys. Lett. B 303, 130 (1993).

[22] S. Chernyshev, M. A. Nowak, and I. Zahed, Phys. Rev. D 53, 5176 (1996).

[23] M. Karliner, J. L. Rosner, and T. Skwarnicki, Annu. Rev. Nucl. Part. Sci. 68, 17 (2018); M. Karliner and J. L. Rosner, Phys. Rev. Lett. 119, 202001 (2017); E. J. Eichten and C. Quigg, Phys. Rev. Lett. 119, 202002 (2017); A. Czarnecki, B. Leng, and M. B. Voloshin, Phys. Lett. B 778, 233 (2018).

[24] M. Karliner and J. L. Rosner, Phys. Rev. Lett. 115, 122001 (2015); M. Karliner, EPJ Web Conf. 130, 01003 (2016).

[25] R. Chen, X. Liu, X. Q. Li, and S. L. Zhu, Phys. Rev. Lett. 115, 132002 (2015); H. X. Chen, W. Chen, X. Liu, T. G. Steele, and S. L. Zhu, Phys. Rev. Lett. 115, 172001 (2015); L. Roca, J. Nieves, and E. Oset, Phys. Rev. D 92, 094003 (2015); T. J. Burns, Eur. Phys. J. A 51, 152 (2015); H. Huang, C. Deng, J. Ping, and F. Wang, Eur. Phys. J. C 76, 624 (2016); L. Roca and E. Oset, Eur. Phys. J. C 76, 591 (2016); Q. F. L and Y. B. Dong, Phys. Rev. D 93, 074020 (2016); Y. Shimizu, D. Suenaga, and M. Harada, Phys. Rev. D 93, 114003 (2016); C. W. Shen, F. K. Guo, J. J. Xie, and B. S. Zou, Nucl. Phys. A954, 393 (2016); M. I. Eides, V. Y. Petrov, and M. V. Polyakov, Phys. Rev. D 93, 054039 (2016); I. A. Perevalova, M. V. Polyakov, and P. Schweitzer, Phys. Rev. D 94, 054024 (2016); V. Kopeliovich and I. Potashnikova, Phys. Rev. D 93, 074012 (2016); Y. Yamaguchi and E. Santopinto, Phys. Rev. D 96, 014018 (2017); S. Takeuchi and M. Takizawa, Phys. Lett. B 764, 254 (2017).

[26] N. N. Scoccola, D. O. Riska, and M. Rho, Phys. Rev. D 92 , 051501 (2015).

[27] M. Z. Liu, Y. W. Pan, F. Z. Peng, M. Sanchez Sanchez, L. S. Geng, A. Hosaka, and M. Pavon Valderrama, Phys. Rev. Lett. 122, 242001 (2019); C. Fernandez-Ramirez, A. Pilloni, M. Albaladejo, A. Jackura, V. Mathieu, M. Mikhasenko, J. A. Silva-Castro, and A. P. Szczepaniak (JPAC Collaboration), Phys. Rev. Lett. 123, 092001 (2019); A. Ali and A. Y. Parkhomenko, Phys. Lett. B 793, 365 (2019).

[28] G. Rossi and G. Veneziano, J. High Energy Phys. 06 (2016) 041.

[29] J. Sonnenschein and D. Weissman, arXiv:1606.02732.

[30] M. Karliner and J. L. Rosner, Phys. Rev. D 95, 114012 (2017); G. Yang and J. Ping, Phys. Rev. D 97, 034023 (2018); K. L. Wang, L. Y. Xiao, X. H. Zhong, and Q. Zhao, Phys. Rev. D 95, 116010 (2017); W. Wang and R. L. Zhu, Phys. Rev. D 96, 014024 (2017); H. Y. Cheng and C. W.
Chiang, Phys. Rev. D 95, 094018 (2017); H. Huang, J. Ping, and F. Wang, Phys. Rev. D 97, 034027 (2018); B. Chen and X. Liu, Phys. Rev. D 96, 094015 (2017); T. M. Aliev, S. Bilmis, and M. Savci, arXiv:1704.03439; H. C. Kim, M. V. Polyakov, and M. Praszalowicz, Phys. Rev. D 96, 014009 (2017).

[31] D. Ebert, R. N. Faustov, and V. O. Galkin, Phys. Rev. D 84, 014025 (2011); Phys. Lett. B 659, 612 (2008); W. Roberts and M. Pervin, Int. J. Mod. Phys. A 23, 2817 (2008).

[32] Z. G. Wang, Eur. Phys. J. C 76, 70 (2016); 77, 325 (2017).

[33] M. Padmanath and N. Mathur, Phys. Rev. Lett. 119, 042001 (2017).

[34] E. V. Shuryak, Nucl. Phys. B198, 83 (1982); N. Isgur and M. B. Wise, Phys. Rev. Lett. 66, 1130 (1991); A. V. Manohar and M. B. Wise, Cambridge Monogr. Part. Phys., Nucl. Phys., Cosmol. 10, 1 (2000).

[35] M. A. Nowak, M. Rho, and I. Zahed, Phys. Rev. D 48, 4370 (1993); Acta Phys. Pol. B 35, 2377 (2004).

[36] W. A. Bardeen and C. T. Hill, Phys. Rev. D 49, 409 (1994); W. A. Bardeen, E. J. Eichten, and C. T. Hill, Phys. Rev. D 68, 054024 (2003).

[37] B. Aubert et al. (BABAR Collaboration), Phys. Rev. Lett. 90, 242001 (2003).

[38] D. Besson et al. (CLEO Collaboration), Phys. Rev. D 68, 032002 (2003); 75, 119908(E) (2007).

[39] J. M. Maldacena, Int. J. Theor. Phys. 38, 1113 (1999); Adv. Theor. Math. Phys. 2, 231 (1998); S. S. Gubser, I. R. Klebanov, and A. M. Polyakov, Phys. Lett. B 428, 105 (1998); E. Witten, Adv. Theor. Math. Phys. 2, 505 (1998); I. R. Klebanov and E. Witten, Nucl. Phys. B556, 89 (1999).

[40] J. Erlich, E. Katz, D. T. Son, and M. A. Stephanov, Phys. Rev. Lett. 95, 261602 (2005); L. Da Rold and A. Pomarol, Nucl. Phys. B721, 79 (2005).

[41] S. Hong, S. Yoon, and M. J. Strassler, J. High Energy Phys. 04 (2006) 003; J. Erlich, G. D. Kribs, and I. Low, Phys. Rev. D 73, 096001 (2006); H. R. Grigoryan and A. V. Radyushkin, Phys. Rev. D 76, 095007 (2007); Phys. Lett. B 650, 421 (2007); S. S. Afonin and I. V. Pusenkov, EPJ Web Conf. 125, 04004 (2016); N. R. F. Braga, M. A. Martin Contreras, and S. Diles, Europhys. Lett. 115, 31002 (2016); A. Gorsky, S. B. Gudnason, and A. Krikun, Phys. Rev. D 91, 126008 (2015).

[42] T. Sakai and S. Sugimoto, Prog. Theor. Phys. 113, 843 (2005); T. Sakai and S. Sugimoto, Prog. Theor. Phys. 114, 1083 (2005).

[43] T. Fujiwara, T. Kugo, H. Terao, S. Uehara, and K. Yamawaki, Prog. Theor. Phys. 73, 926 (1985).

[44] Y. Liu and I. Zahed, Phys. Rev. D 95, 056022 (2017); Phys. Lett. B 769, 314 (2017); Phys. Rev. D 95, 116012 (2017).

[45] S. w. Li, Phys. Rev. D 96, 106018 (2017); W. Cai and S. w. Li, Eur. Phys. J. C 78, 446 (2018); S. w. Li, Phys. Rev. D 99, 046013 (2019).

[46] H. Hata, T. Sakai, S. Sugimoto, and S. Yamato, Prog. Theor. Phys. 117, 1157 (2007).

[47] K. Hashimoto, T. Sakai, and S. Sugimoto, Prog. Theor. Phys. 120, 1093 (2008); K. Y. Kim and I. Zahed, J. High Energy Phys. 09 (2008) 007.

[48] H. Hata and M. Murata, Prog. Theor. Phys. 119, 461 (2008).

[49] K. Hashimoto, N. Iizuka, T. Ishii, and D. Kadoh, Phys. Lett. B 691, 65 (2010). 
[50] P. H. C. Lau and S. Sugimoto, Phys. Rev. D 95, 126007 (2017).

[51] I. Zahed and G. E. Brown, Phys. Rep. 142, 1 (1986); Multifaceted Skyrmion, edited by M. Rho and I. Zahed (World Scientific, Singapore, 2016).

[52] N. N. Scoccola, Nucl. Phys. A532, 409 (1991); M. Rho, D. O. Riska, and N. N. Scoccola, Z. Phys. A 341, 343 (1992); D. P. Min, Y. s. Oh, B. Y. Park, and M. Rho, arXiv: hep-ph/9209275; Y. s. Oh, B. Y. Park, and D. P. Min, Phys. Rev. D 49, 4649 (1994); 50, 3350 (1994); D. P. Min, Y. s. Oh, B. Y. Park, and M. Rho, Int. J. Mod. Phys. E 04, 47 (1995); Y. s. Oh and B. Y. Park, Phys. Rev. D 51, 5016 (1995); J. Schechter, A. Subbaraman, S. Vaidya, and H. Weigel, Nucl. Phys. A590, 655 (1995); 598, 583(E) (1996); Y. S. Oh and B. Y. Park, Z. Phys. A 359, 83 (1997); C. L. Schat and N. N. Scoccola, Phys. Rev. D 61, 034008 (2000); N. N. Scoccola, arXiv:0905.2722; N. Itzhaki, I. R. Klebanov, P. Ouyang, and L. Rastelli, Nucl. Phys. B684, 264 (2004); J. P. Blanckenberg and H. Weigel, Phys. Lett. B 750, 230 (2015); M. Praszalowicz, Proc. Sci., CORFU2017 (2018) 025 [arXiv:1805.07729].

[53] A. Paredes and P. Talavera, Nucl. Phys. B713, 438 (2005); J. Erdmenger, N. Evans, and J. Grosse, J. High Energy Phys. 01 (2007) 098; J. Erdmenger, K. Ghoroku, and I. Kirsch, J. High Energy Phys. 09 (2007) 111; C. P. Herzog, S. A. Stricker, and A. Vuorinen, J. High Energy Phys. 05 (2008) 070; Y. Bai and H. C. Cheng, J. High Energy Phys. 08 (2013) 074; K. Hashimoto, N. Ogawa, and Y. Yamaguchi, J. High Energy Phys. 06 (2015) 040.

[54] G. F. de Teramond, S. J. Brodsky, A. Deur, H. G. Dosch, and R. S. Sufian, EPJ Web Conf. 137, 03023 (2017); H. G. Dosch, G. F. de Teramond, and S. J. Brodsky, Phys. Rev. D 92, 074010 (2015); H. G. Dosch, G. F. de Teramond, and S. J. Brodsky, Phys. Rev. D 95, 034016 (2017).
[55] J. Sonnenschein and D. Weissman, Nucl. Phys. B920, 319 (2017); J. Sonnenschein and D. Weissman, Eur. Phys. J. C 79, 326 (2019).

[56] M. Luscher, Phys. Lett. 70B, 321 (1977); B. M. Schechter, Phys. Rev. D 16, 3015 (1977).

[57] D. M. Ostrovsky, G. W. Carter, and E. V. Shuryak, Phys. Rev. D 66, 036004 (2002).

[58] E. Shuryak and I. Zahed, Phys. Rev. D 67, 014006 (2003).

[59] F. Brunner, D. Parganlija, and A. Rebhan, Phys. Rev. D 91, 106002 (2015); 93, 109903(E) (2016).

[60] K. M. Case, Phys. Rev. 80, 797 (1950); S. R. Beane, P. F. Bedaque, L. Childress, A. Kryjevski, J. McGuire, and U. van Kolck, Phys. Rev. A 64, 042103 (2001); E. Braaten and D. Phillips, Phys. Rev. A 70, 052111 (2004).

[61] V. Efimov, Phys. Lett. 33B, 563 (1970).

[62] A. Francis, R. J. Hudspith, R. Lewis, and K. Maltman, Phys. Rev. D 99, 054505 (2019).

[63] G. K. C. Cheung, C. E. Thomas, J. J. Dudek, and R. G. Edwards (Hadron Spectrum Collaboration), J. High Energy Phys. 11 (2017) 033.

[64] M. J. Savage and M. B. Wise, Phys. Lett. B 248, 177 (1990).

[65] C. G. Callan and I. Klebanov, Nucl. Phys. B262, 365 (1985); For a review, see e.g., M. A. Nowak, M. Rho, and I. Zahed, Nuclear Chiral Dynamics (World Scientific, Singapore, 1996).

[66] Y. R. Liu, H. X. Chen, W. Chen, X. Liu, and S. L. Zhu, Prog. Part. Nucl. Phys. 107, 237 (2019).

[67] U. Gursoy and E. Kiritsis, J. High Energy Phys. 02 (2008) 032; U. Gursoy, E. Kiritsis, and F. Nitti, J. High Energy Phys. 02 (2008) 019. 